\title{
Interactions between vortex tubes and magnetic-flux rings at high kinetic and magnetic Reynolds numbers
}

\author{
Demosthenes Kivotides \\ University of Strathclyde Glasgow
}

(Dated: February 8, 2018)

\begin{abstract}
The interactions between vortex tubes and magnetic-flux rings in incompressible MHD are investigated at high kinetic and magnetic Reynolds numbers, and over a wide range of the interaction parameter. The latter is a measure of the turnover time of the large-scale fluid motions in units of the magnetic damping time, or of the strength of the Lorentz force in units of the inertial force. The small interaction parameter results, that are related to kinematic turbulent dynamo studies, indicate the evolution of magnetic-rings into flattened spirals wrapped around the vortex tubes. This process is also observed at intermediate interaction parameter values, only now the Lorentz force creates new vortical structures at the magnetic spiral edges, that have a striking solenoid vortex-line structure, and endow the flattened magnetic-spiral surfaces with a curvature. At high interaction parameter values, the decisive physical factor is Lorentz force effects. The latter create two (adjacent to the magnetic-ring) vortex rings, that reconnect with the vortex tube by forming an intriguing, serpentine-like, vortex-line structure, and generate, in turn, two new magnetic rings, adjacent to the initial one. In this regime, the morphologies of the vorticity and magnetic field structures are similar. The effects of these structures on kinetic and magnetic energy spectra, as well as on the direction of energy transfer between flow and magnetic fields are also indicated.
\end{abstract}




\section{PROLOGUE}

Turbulence in fluids is a key problem in classical [1], statistical [2], quantum [3, 4], and relativistic [5, 6] physics. In contrast to random/Gaussian fluctuations, turbulence is characterized by non-equilibrium, interscale energy transfer processes, which in (incompressible fluids) give rise to non-Gaussian fluctuations, as exemplified by the non-vanishing third order structure function of the main dynamical field, the flow velocity [7, 8]. Perhaps an even more striking characteristic of turbulent chaos, is the key physical role of the curl of velocity, i.e., the flow vorticity. The latter is characterized by coherent/metastable structures (tubes and sheets), which are an example of spontaneous dynamical long-range order [9], and are embedded amidst regions of fluctuating, smaller magnitude vorticity. The dynamics of coherent vortical structures contribute heavily to the statistical phenomenology of turbulence (e.g., there is an association between vortex stretching and energy cascade), yet, due to the analytical intractability of the strong turbulent nonlinearity, it has not been possible until now to analytically understand their key interactions and, via averaging, to incorporate the latter into the various statistical theories of turbulence. Another important characteristic of incompressible fluids is the absence of any wave phenomena, since (by default) there are no sound waves in the system. The above discussion applies to the hydrodynamics of classical (potential) field theories, e.g., particle systems interacting via the Lennard-Jones force, where the force-potential does not appear explicitly in the hydrodynamics (albeit hidden within the fluid stress tensor). This feature is not necessarily a consequence of the potential character of the interaction force, and indeed the hydrodynamics of Chromodynamic Plasmas $[10,11]$ can also be modeled as relativistic fluids, without any reference to the microscopic, relativistic gauge field. It is in this context, that the study of Electrodynamic Plasmas [12] becomes a very interesting area of turbulence theory, since (a) the gauge (magnetic) field explicitly appears in the hydrodynamics (hence, magnetohydrodynamics $[\mathrm{MHD}])$, and, via its convection by the velocity field, becomes turbulent, developing coherent structures of its own. Thus, it is conceptually important to understand the interactions between structures in the gauge and inertial fields, and the way these can help understand (in a structural way) some of the complexity of turbulent chaos, (b) the presence of the Lorentz force in the Navier-Stokes equations enables the depiction of wave phenomena in the latter (Alfven waves), which lead to novel (in comparison with incompressible turbulence) 
phenomenology, such as the propagation of transverse inertial waves along magnetic field lines.

In standard textbooks [13], the kinematic analogy between velocity/vorticity on the one hand and magnetic field/current on the other is stressed. However, from the dynamic point of view, it is better to draw an analogy (in the indicated order) between the three inertial fields $(\mathbf{u}, \boldsymbol{\omega}, \boldsymbol{\xi})$, where $\mathbf{u}$ is the flow velocity, $\boldsymbol{\omega}=\nabla \times \mathbf{u}$ is the flow vorticity, and $\boldsymbol{\xi}=\nabla \times \boldsymbol{\omega}$ is the flow palin-vorticity, and the three gauge theoretic fields $(\mathbf{A}, \mathbf{B}, \mathbf{J})$, where $\mathbf{A}$ is the electromagnetic vector potential, $\mathbf{B}=\nabla \times \mathbf{A}$ is the magnetic field, and $\mathbf{J}=\nabla \times \mathbf{B} / \mu$ is the electric current (and $\mu$ the permeability of free space). In this way, $\mathbf{B}$ corresponds to $\boldsymbol{\omega}$. Indeed, B obeys a similar equation with that of $\boldsymbol{\omega}$, which allows for local evolution that preserves any structural field aspects, in opposition to $\mathbf{u}$ that is subjected to nonlocal pressure effects that destroy coherent velocity patterns. This analogy holds in the definitions of quantities like kinetic helicity $\mathrm{H}^{\mathrm{K}}=\mathbf{u} \cdot \boldsymbol{\omega}$, the corresponding magnetic helicity $\mathrm{H}^{\mathrm{M}}=\mathbf{A} \cdot \mathbf{B}$, and cross helicity $\mathrm{H}^{\mathrm{C}}=\mathbf{u} \cdot \mathbf{B}$, but it is not complete since, for example, in the NavierStokes equation, the Lamb force term $\boldsymbol{\omega} \times \mathbf{u}$ would have been paired with a term $\mathbf{B} \times \mathbf{A}$, whilst, instead, the Lorentz force $\mathbf{J} \times \mathbf{B}$ appears. This difference in form reflects upon more fundamental physics differences, since the Lamb force (been part of the inertial force) is conservative, and responsible for the generation of the complexity we call turbulence, whilst the Lorentz force simply smooths out turbulent motions via the $\mathbf{J}^{2} / \sigma$ term (and governs energy transfer between magnetic and velocity fields via the $\mathbf{J} \cdot \mathbf{E}$ term). Moreover, it is important to note that, although the transport of $\mathbf{B}$ is similar to the transport of $\boldsymbol{\omega}$, the evolution histories available to $\mathbf{B}$ are a superset of those available to $\boldsymbol{\omega}$, since $\boldsymbol{\omega}$ is the curl of the convective field that does the transport, a constraint that does not bind the dynamics of $\mathbf{B}$. Having in mind these (important) caveats, in this article, we employ $\mathbf{B}$ and $\boldsymbol{\omega}$ as the most appropriate pair of gauge and inertial fields whose structural interactions can lead to a "synthetic" understanding of more complicated MHD turbulence processes. In doing so, we follow a long tradition in turbulence theory [14-17], that is complementary to other statistical approaches [18, 19]. Inspired by fully resolved Navier-Stokes turbulence results, we choose a straight vortex tube as a rough model of turbulence structure. Indeed, as shown in Fig.1 (left), the isosurfaces of enstrophy in homogeneous, isotropic, Navier-Stokes turbulence of Taylor Reynolds number $R e_{\lambda} \approx 100$ show many linear structures of weak 

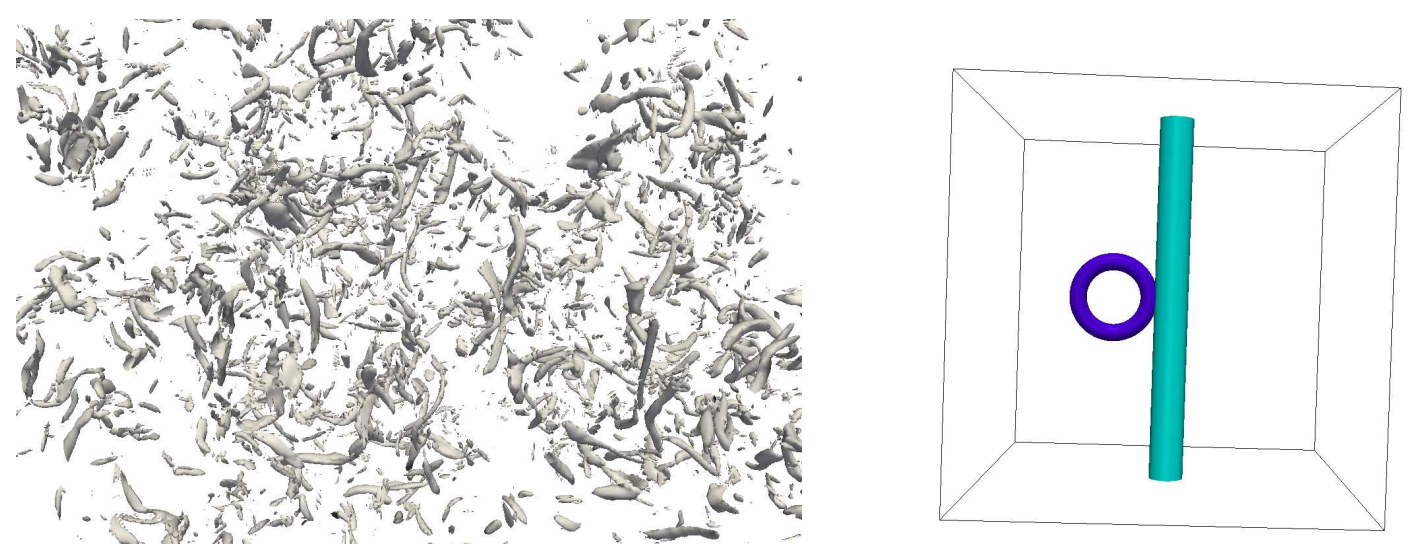

FIG. 1. Left: Vorticity magnitude isosurfaces at level equal to 0.38 times its maximum value, for a homogeneous, isotropic, incompressible Navier-Stokes turbulence at Taylor Reynolds number $R e_{\lambda} \approx 100$. Results taken from an accompanying incompressible (non MHD) turbulence calculation. Although sheet-like structures are present, we predominantly see linear vortices. Right: The initial configuration consists of a straight vortex tube, and a magnetic-flux ring, in a periodic box. Both isosurfaces correspond to levels equal to half of the corresponding maximum field-magnitude values.

curvature. Moreover, calculations of MHD turbulence [20] have indicated the importance of interactions between strong vortex tubes and magnetic flux tubes, having the latter wrap around the former, increasing their curvature. Employing Vortex Dynamics methods, [21] have explicitly depicted similar processes in a kinematic turbulent dynamo computation, that analyzed the effect of filamentary vorticity structures on a seed, random magnetic field. It was shown, that the magnetic field intensifies in between the vortex filaments, and, as it forms thick, ribbon-like structures, it is wrapped around them. Although interactions between straight $\boldsymbol{\omega}$ and $\mathbf{B}$ tubes are important, we choose here to study magnetic-flux rings, since, the latter introduce (all important) curvature effects, as recognized in earlier publications [22]. Magnetic rings are key MHD structures. Indeed, [23] suggested that rings produced by the collision of two solitary magnetic kinks along a straight magnetic flux tube play a role in solar flare formation, and [24] have computed the dynamics of linked magnetic rings in order to determine the role of magnetic helicity in magnetic field decay. For the case of magnetic tangles/knots with zero net magnetic helicity, [25] have shown that such structures are unstable, and split into two packages moving in opposite directions, each with finite and opposite magnetic helicity. 
In a more general turbulence context, tubes and rings are examples of coherent structures. The latter have been intensively studied in MHD turbulence, including their generation via flow instabilities [26], their detection [27], the role of compressibility in their dynamics [28], as well as, their effects on stochastic particle acceleration [29], and energy dissipation [30]. One of the goals of the present investigation is to help understand better these findings of fully resolved turbulence calculations, by modeling coherent structures explicitly, and with fine resolution, so that it would be possible to identify some of the complex phenomenology of turbulence within the present explicit flow patterns. Our initial conditions are nonhelical, and our magnetic Prandtl number $\left(\operatorname{Pr}_{m}=\nu / \lambda\right.$, where $\nu$ is the kinematic viscosity and $\lambda$ the magnetic diffusivity) is unity, hence the results are related to nonhelical, unity Prandtl number turbulence calculations [31] showing organization of the magnetic field in the form of flux tubes, and magnetic sheets. Coherent structure studies with large $\operatorname{Pr}_{m}$ values would relate to corresponding turbulence computations [32], and could be performed in the future.

\section{MATHEMATICAL MODEL}

We analyze the standard (non Hall [33]) incompressible MHD system, that includes the fluid momentum equation,

$$
\frac{\partial \mathbf{u}}{\partial t}+\nabla\left(\frac{p}{\rho}+\frac{\mathbf{u} \cdot \mathbf{u}}{2}+\frac{\mathbf{B} \cdot \mathbf{B}}{2 \rho \mu}\right)-\mathbf{u} \times \boldsymbol{\omega}-\frac{1}{\rho \mu}(\mathbf{B} \cdot \nabla) \mathbf{B}-\nu \nabla^{2} \mathbf{u}=0
$$

the equation for the magnetic field transport,

$$
\frac{\partial \mathbf{B}}{\partial t}+(\mathbf{u} \cdot \nabla) \mathbf{B}-(\mathbf{B} \cdot \nabla) \mathbf{u}-\lambda \nabla^{2} \mathbf{B}=0
$$

and the two elliptic constraints, $\nabla \cdot \mathbf{u}=0$, and $\nabla \cdot \mathbf{B}=0$, that enforce the solenoidal character of $\mathbf{u}$ and $\mathbf{B}$. Here, $p$ is the fluid pressure field, and $\rho$ is the fluid density. Notably, the above system could be neatly written in terms of the "velocity" $\widetilde{\mathbf{B}}=\mathbf{B} / \sqrt{\rho \mu}$. In the following, we shall denote (for example) by $\widetilde{\mathbf{J}}$ or $\widetilde{\Psi}$, electrodynamic quantities incorporating the above scaling for $\mathbf{B}$. It is also helpful to write the transport equation for $\boldsymbol{\omega}$

$$
\frac{\partial \boldsymbol{\omega}}{\partial t}+(\mathbf{u} \cdot \nabla) \boldsymbol{\omega}-(\boldsymbol{\omega} \cdot \nabla) \mathbf{u}-\frac{1}{\rho}[(\mathbf{B} \cdot \nabla) \mathbf{J}-(\mathbf{J} \cdot \nabla) \mathbf{B}]-\nu \nabla^{2} \boldsymbol{\omega}=0,
$$

where, we observe that the reaction of the magnetic field on the fluid introduces an electrodynamic term $\nabla \times(\mathbf{J} \times \mathbf{B})$, that can act as a vorticity source, differentiating, in this way, 
the $\boldsymbol{\omega}$ and $\mathbf{B}$ evolution laws. The $\boldsymbol{\omega}$ and $\mathbf{u}$ fields are kinematically related via the Poisson equation

$$
\nabla^{2} \mathbf{u}+\nabla \times \boldsymbol{\omega}=0
$$

It is helpful to note that, under reasonable boundary conditions (that include periodicity), the $\mathcal{V}_{\infty}$ integrals of the kinetic, magnetic and cross helicities (defined above) are inviscid invariants in MHD. Hence, in the viscous calculations presented here, they are expected to vary on the diffusion time scales only, since their values are conserved by convective motions $[12,18,34]$. An intuitive understanding of helicities as inviscid invariants follows from the facts that $H^{K}, H^{M}, H^{C}$ inform about the self-linkage or knottedness (topology) of vortex, magnetic or vortex/magnetic tubes correspondingly, and that vortex and magnetic-field lines have a "frozen-in" character in ideal flows. Indeed, since topological change occurs via flux-tube reconnections that rely on viscous action in order to allow the vortex/magnetic lines to "slip" relative to the velocity field, it follows that the "frozen-in" lines of ideal hydrodynamics have no means of altering their topology, hence their helicities ought to be preserved. In other words, helicity plays the role of a topological charge [35, 36].

Finally, we define (a) the flux of vorticity along a vortex tube $\Phi$,

$$
\Phi=\int \boldsymbol{\omega} \cdot \mathrm{d} \mathbf{S}=\oint \mathbf{u} \cdot \mathrm{d} \mathbf{l}
$$

where the first integral is over the cross sectional area of a vortex tube, and the second integral (which follows from Stokes Theorem) is the circulation of $\mathbf{u}$ around the tube, and (b) the magnetic flux along a magnetic-field tube $\Psi$,

$$
\Psi=\int \mathbf{B} \cdot \mathrm{d} \mathbf{S}=\oint \mathbf{A} \cdot \mathrm{d} \mathbf{l}
$$

where the first integral is over the cross sectional area of a magnetic tube, and the second integral (which follows from Stokes Theorem) is the circulation of $\mathbf{A}$ around the tube. $\Phi$ and $\Psi$ are going to be referred to as "tube strengths" in the following.

\section{SOLUTION METHODS}

The (strongly nonlinear) mathematical model is solved via a staggered grid, fractional step, projection, finite volume method [37, 38]. A book-length discussion of the method 
is available in [39]. All spatial partial derivatives are computed with second order accurate schemes. The method employs an explicit, third order accurate in time, low storage Runge-Kutta (RK) method for the computation of advective and source terms. An implicit, second order accurate in time Crank-Nicolson $(\mathrm{CN})$ scheme is applied to the viscous/diffusion terms. Since CN scheme is implicit and the boundary conditions are periodic, the method requires the solution of cyclic-tridiagonal, linear algebraic equation systems. We have used the Sherman-Morrison formula in order to reduce the latter to much easily solved tridiagonal systems. The CN scheme is incorporated into the RK steps and the method becomes a hybrid RK/CN scheme. In other words, the viscous stress terms are advanced via the CN scheme, but within the three Runge-Kutta time substeps rather than in one large time step.

In the method, the sum of fluid $p$ and magnetic $\frac{\mathbf{B} \cdot \mathbf{B}}{2 \rho \mu}$ pressures define an effective pressure whose gradient requires special treatment, in order to avoid instabilities. It is incorporated explicitly into the RK steps, but in order to enforce incompressibility, an additional calculation after each RK substep projects the velocity field onto the space of divergence-free vector fields (Hodge projection) [39]. The latter computation is equivalent to a separate velocity upgrade due to the effective pressure gradient. The net result of RK and Hodge projection procedures is that, depending on the chosen scheme parameters, pressure gradient effects are captured with first or second order in time accuracy. In agreement with the literature [39], we have found the first order accurate in time scheme to be more stable, and we have employed it here. The incompressibility of the magnetic field, is also enforced, at every time step, via Hodge projection. On the algorithmics side, the computation of the RHS (i.e., of all terms excepting the time derivative) of velocity and magnetic field equations is performed first, followed by time advancement, and, as a final processing step, by the Hodge projection of velocity and magnetic fields.

\section{INITIAL, BOUNDARY CONDITIONS AND CALCULATION SPECIFICS}

The initial conditions include a straight vortex tube, and a magnetic-flux ring within a box of size $l_{b}$ (Fig.1, right). Both $\boldsymbol{\omega}$ and $\mathbf{B}$ structures are prescribed following the axisymmetric model of [40]. The tube axis belongs to the plane of the ring. Indeed, if the tube extends along the $z$ axis, then the ring is placed on the $x z$ plane, and its center is located at the $\left(-0.16 l_{b}, 0,0\right)$ point. As mentioned in the Prologue, this configuration, in conjunction 
with the axisymmetric distribution of both $\boldsymbol{\omega}$ and $\mathbf{B}$, makes the initial conditions nonhelical (with respect to all three helicity types). The vorticity and magnetic fields follow a Gaussian distribution within the tube/ring, and the cross-sectional radii of the latter (defined via the standard deviation of the distribution function) are equal to $0.04 l_{b} / 0.02 l_{b}$ respectively. Since the radius of the magnetic flux tube is equal to $0.1 l_{b}$, it follows that the ring's cross section has a distance equal to $0.16-0.1-0.02=0.04 l_{b}$ from the tube axis, hence, it exactly touches upon the cross section of the vortex.

We do three calculations with same kinetic Reynolds number $R e_{k}=u \ell / \nu$, where $u$ is a characteristic velocity, and $\ell$ a characteristic length scale (that can be taken to be of the order of the cross sectional tube/ring radius, so we choose $\ell=0.02 l_{b}$ ). Since $P r_{m}=1$, the magnetic Reynolds number $R e_{m}=u \ell / \lambda$ is equal to $R e_{k}$. We adopt a "vortex dynamical" definition of $R e_{k}$ : since $\Phi$ has $u \ell$ units, it follows that $\Phi / \nu$ is an effective kinetic Reynolds number, which is all calculations is set to $R e_{k}=10^{4}$. Since this is also $R e_{m}$, how are we to understand the three different computations presented here? It is best to begin by recalling the concept of the interaction parameter $N=\nabla \times($ Lorentz force $) / \nabla \times($ inertial force $)$, which scales the Lorentz force against the inertial force, hence, it indicates how important the magnetic field's action on the flow is [7]. Here, we write $N=\sigma B^{2} \ell / \rho u$ where $B$ is a characteristic magnetic field magnitude, and $\sigma$ is the electrical conductivity. Notably, this formula for $N$ involves only one characteristic length scale $\ell$ for the velocity variations (same as in the $R e$ number formula), hence, it implicitly assumes that the characteristic velocity variation length scales normal and parallel to the magnetic field within the initial flux-tube are similar. The excellent agreement between the scaled $N$ values and the corresponding physics in the numerical solutions justify this assumption. Since $N$ scales with the square of the characteristic magnetic field magnitude, it follows that a nice way of controlling $N$ is by tuning the magnetic flux $\Psi$. Indeed, using $\lambda=(\mu \sigma)^{-1}$, we have $N=\sigma \mu B^{2} \ell^{2} / \rho \mu u \ell=\nu \widetilde{B}^{2} \ell^{2} / u \ell \nu \lambda=\nu \widetilde{B}^{2} \ell^{4} / u \ell \nu \lambda \ell^{2}$, and using $\widetilde{\Psi} \approx \widetilde{B} \ell^{2}$, we get $N=\widetilde{\Psi}^{2} / R e_{k} \nu \lambda \ell^{2}=\widetilde{\Psi}^{2} / R e_{k}(\lambda \ell)^{2}$, since $P r_{m}=1$. In terms of the interaction parameter then, we do three calculations, with $N_{1}=2.510^{-3}, N_{2}=2.510$, and $N_{3}=2.510^{3}$, that intuitively correspond to very small (kinematic dynamo regime, as in [21]), significant, and very strong Lorentz force effects on the flow (with special emphasis on the generation of vorticity).

The periodicity was enforced by considering the effects on $\mathbf{u}$ and $\mathbf{A}$ of vortex tubes and magnetic-flux rings in all adjacent boxes. The numerics allowed stable computations with 
Courant-Friedrichs-Lewy (CFL) number, $\mathrm{CFL}=0.75$ (for both velocity and magnetic fields). Velocity incompressibility was enforced with typical accuracy $10^{-10}$, and magnetic-field incompressibility with typical accuracy $10^{-12}$. The time step is chosen so that the viscous/diffusion time-scales are resolved. In $N_{1}$ and $N_{2}$ cases, the time step was dictated by $\mathbf{u}$ evolution, whilst, in the $N_{3}$ case, sometimes $\mathbf{u}$ and sometimes $\mathbf{B}$ evolutions have restricted the computational timestep. However, in all cases, the time step was dictated by the CFL stability criterion, whose corresponding time steps (typically) were an order of magnitude smaller than the viscous/diffusion time steps. A fine $512^{3}$ staggered grid was employed throughout. Due to the high $R e_{m}$ value, the results are more relevant to astronomical, rather than technological or geological plasmas. Indeed, within the liquid core of the earth, the magnetic Reynolds number of large eddies is $R e_{m} \approx 100$ [7], whilst in liquid-metals at scales characteristic of laboratory or industrial settings, $R e_{m}$ is significantly smaller than unity [41].

The calculations are expected to indicate significant dynamics of vorticity and magnetic field structures due to their interactions with flow strain. Indeed, from many important points of view, the basic field in fluid dynamics is not a vector (i.e., the velocity $\mathbf{u}$, written in component form as $u_{i}$, and similarly for the components $\omega_{i}$ of vorticity), but a tensor (velocity gradient tensor $A_{i j}=\partial u_{i} / \partial x_{j}$ ). This is because $A_{i j}=S_{i j}-\frac{1}{2} \epsilon_{i j k} \omega_{k}$, so $A_{i j}$ encodes both rate of strain $\left(S_{i j}=\frac{1}{2}\left(\partial u_{i} / \partial x_{j}+\partial u_{j} / \partial x_{i}\right)\right)$ and solid-body rotation local aspects of fluid matter. These aspects and their interactions form the basic vocabulary of fluid flow analysis. To start, it is important to know the three (ordered) eigenvalues $\lambda_{i}$ and corresponding eigenvectors $\boldsymbol{\lambda}_{i}$ of $S_{i j}$. This is because they give direct information about the nature of local flow (i.e., uniaxial or biaxial extensional flow) and the alignments between straining process and vorticity and magnetic field vectors. Based on these key quantities, some important physical measures can be established [8]. First, the vortex stretching vector $W_{i}=\omega_{i} S_{i j}$, whose internal product with vorticity gives the enstrophy source (amplification in turbulence) $\omega_{i} \omega_{j} S_{i j}$. Similarly, there is a magnetic stretching vector $M_{i}=B_{i} S_{i j}$, whose internal product with magnetic field gives the magnetic energy source (amplification in turbulence) $B_{i} B_{j} S_{i j}$. Hence, key quantities for understanding enstrophy production are the cosines $\cos \left(\boldsymbol{\omega}, \boldsymbol{\lambda}_{i}\right)$ and $\cos (\boldsymbol{\omega}, \mathbf{W})$, whilst the corresponding quantities for magnetic energy production are the cosines $\cos \left(\mathbf{B}, \boldsymbol{\lambda}_{i}\right)$ and $\cos (\mathbf{B}, \mathbf{M})$. On the other hand, the (normalized with the square root of the mean total strain $\left\langle S_{i j} S_{i j}\right\rangle^{1 / 2}$ ) mean values of the rate of 
strain eigenvalues $\left\langle\lambda_{i}\right\rangle$ (and similarly the normalized $\left\langle\lambda_{i}^{2}\right\rangle$ values) are indicative of whether extensional or compressive processes characterize (on average) the flow. Moreover, the $\lambda^{\star}$ parameter of Lund and Rogers [42], $\lambda^{\star}=-3 \sqrt{6} \lambda_{1} \lambda_{2} \lambda_{3} /\left(\lambda_{1}^{2}+\lambda_{2}^{2}+\lambda_{3}^{2}\right)^{3 / 2}$, ranges from -1 to 1 , and can also indicate the type of deformations caused by the strain-rate tensor, since $\lambda^{\star}=-1$ corresponds to axisymmetric contraction, $\lambda^{\star}=0$ to planar shear, and $\lambda^{\star}=1$ to axisymmetric extension. The probability density function (pdf) of $\lambda^{\star}$ is uniform for a Gaussian velocity field, whilst, for isotropic turbulence, Lund and Rogers showed that the most probable strain process is axisymmetric extension that is associated with high dissipation flow regions. We shall employ such indicators in order to characterize the flow/gauge-field interactions at different interaction parameter values.

COMPUTATIONAL SOLUTION FOR $R e_{k}=R e_{m}=10^{4}, N=2.5 \times 10^{-3}$

A good measure of time is in units of the viscous/diffusion time-scales of the computation. Since, $\nu=\lambda$, and the numerical grid is identical for both $\mathbf{u}$ and $\mathbf{B}$, viscous flow time-scales and diffusion magnetic time-scales are identical, and equal to $\tau_{d}=(\Delta x)^{2} /(6 \nu)$. Here, $\Delta x$ is the computational grid size. In order to understand the physics, it is important to note that, (a) since, $R e_{m} \gg 1$, and $N \ll 1$, dissipative effects are small, and the tube is not significantly affected by the magnetic field, hence, we expect that the ring is predominantly going to be advected by an undisturbed vortex flow field, (b) that flow ought to remain nonhelical, and (c) the fluid within the tube radius rotates like a solid body, whilst the flow in the region outside is a potential vortex flow, where fluid layers closer to the tube move faster than those far away. As a result, fluid layers rub against each other, and a material line (in the radial direction) tends to become a spiral. Indeed, as shown in Fig.2 (left), the ring is wrapped into a spiral (that at final time has turned four times). In the same figure (right), we observe that the vortex lines within the tube remain straight, and that no significant vorticity has been generated in the ring region. The results agree very well with the vortex dynamical kinematic dynamo in [21]. Fig.3 (left) shows the magnetic field spiral $\left(t=106.25 \tau_{\mathrm{d}}\right.$ ), with superposed magnetic field lines. It is observed that the higher magnetic field magnitudes are located within the flattened-ring structures, where the $\mathbf{B}$ lines are smooth, without any direction-reversals. This morphology is in agreement with the scaled strain-rate eigenvalue results of Table I, that indicate two (on average) positive eigenvalues, hence, due to biaxial 

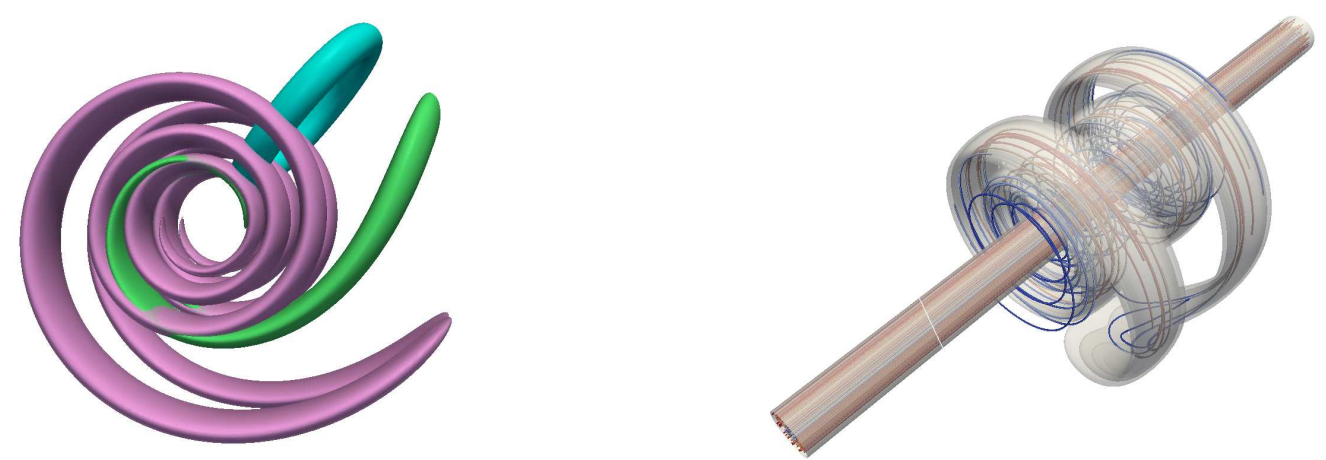

FIG. 2. $\mathrm{N}=2.5 \times 10^{-3}$. Left: Magnetic-flux ring configurations for three different times: $t=$ 0 (undisturbed ring), $t=31.45 \tau_{\mathrm{d}}$ (intermediate ring, just started forming a spiral), and $t=$ $106.25 \tau_{\mathrm{d}}$ (ring warped into a spiral structure). Here, $t=0$ graph simultaneously shows isosurfaces for five values spanning the range $0.5-1$ of maximum magnetic field magnitude, $t=31.45 \tau_{\mathrm{d}}$ graph simultaneously shows isosurfaces for five values spanning the range $0.55-1$ of maximum magnetic field magnitude, and $t=106.25 \tau_{\mathrm{d}}$ graph simultaneously shows isosurfaces for eight values spanning the range $0.8-1$ of maximum magnetic field magnitude. Right: Tube-ring configuration at $t=106.25 \tau_{\mathrm{d}}$ : the ring is wrapped around a, virtually undisturbed, vortex tube, with straight vortex lines. For the magnetic field, isosurfaces for ten values spanning the whole range between minimum and maximum magnetic field magnitudes are shown simultaneously, whilst for the vorticity field, a single isosurface corresponding to half of the maximum vorticity magnitude is shown. Moreover, vortex and magnetic field lines (that are computed via the RungeKutta-Fehlberg numerical integration method) are also depicted. The field-lines color code is the same for both vorticity and magnetic-field lines. Smaller magnitudes correspond to blue color, and larger magnitudes to red color.

extension and strong compression along the third direction, sheet-like magnetic structures are expected. Two positive eigenvalues are also the signature of fully developed turbulence (Table I), however in the latter case, the intermediate eigenvalue is an order of magnitude larger than in our flow. This is also the case for the average eigenvalue squares. The above reflect the fact that turbulent vortex structure is certainly more complicated than an ensemble of straight tubes. The highest magnetic-field line curvature is observed at the locations corresponding to the two parts of the ring (initially) closer and further away from the tube, with the former situated at the innermost part of the spiral. 


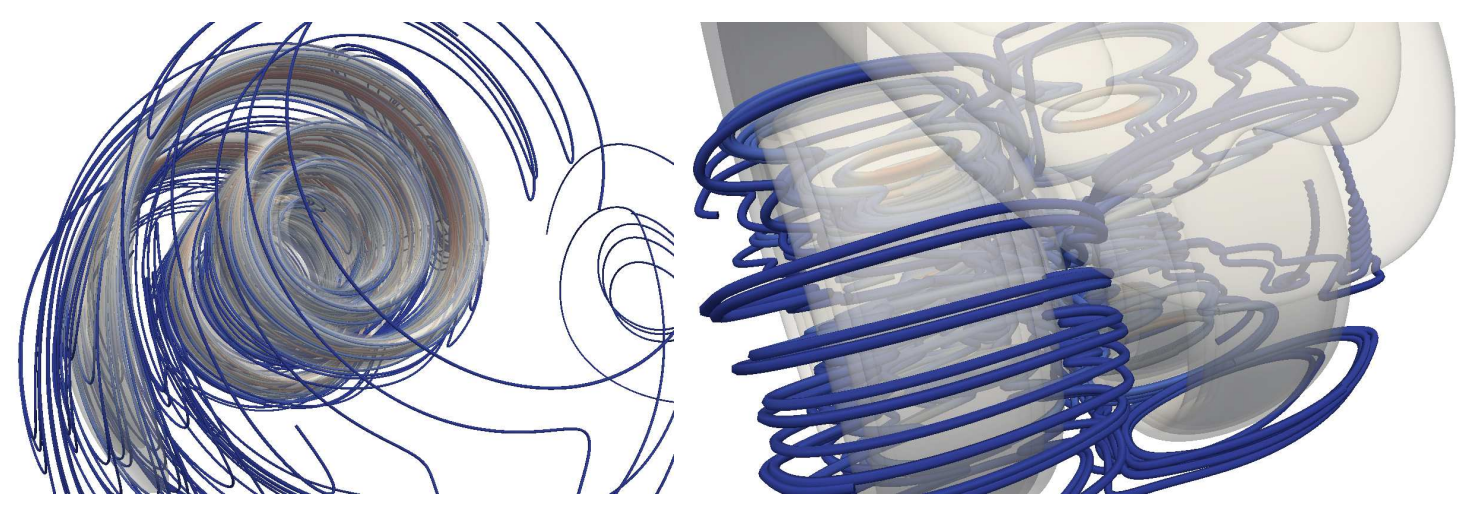

FIG. 3. $\mathrm{N}=2.5 \times 10^{-3}, t=106.25 \tau_{\mathrm{d}}$. Left: Magnetic field spiral, and superposed field-lines. Highest field curvature is associated with the, initially, closest and furthest from the vortex tube parts of the ring, and highest field-magnitude values are located within the flattened arms of the spiral. Right: Magnetic-field isosurfaces (low opacity), and current-lines: relatively weak current engulfs the magnetic field structure, and the highest current values are associated with current loops formed within the structure. In both figures, isosurfaces for ten values spanning the whole range between minimum and maximum magnetic field magnitudes are shown simultaneously. The field-lines are computed via the Runge-Kutta-Fehlberg numerical integration method.

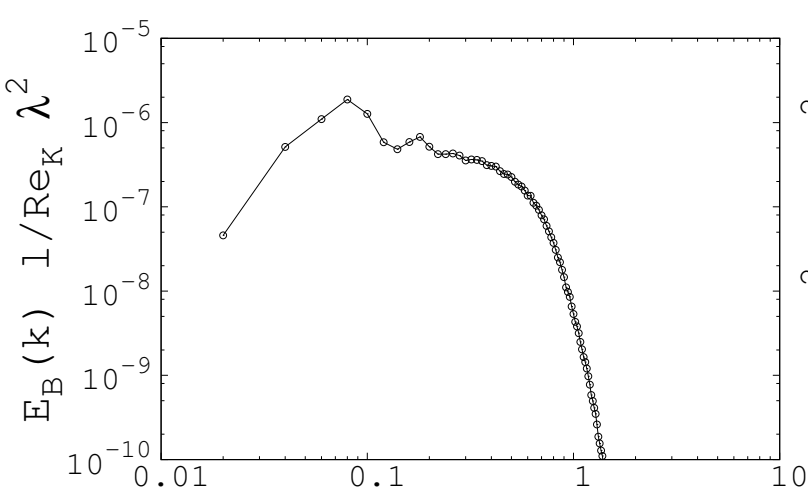

k 1

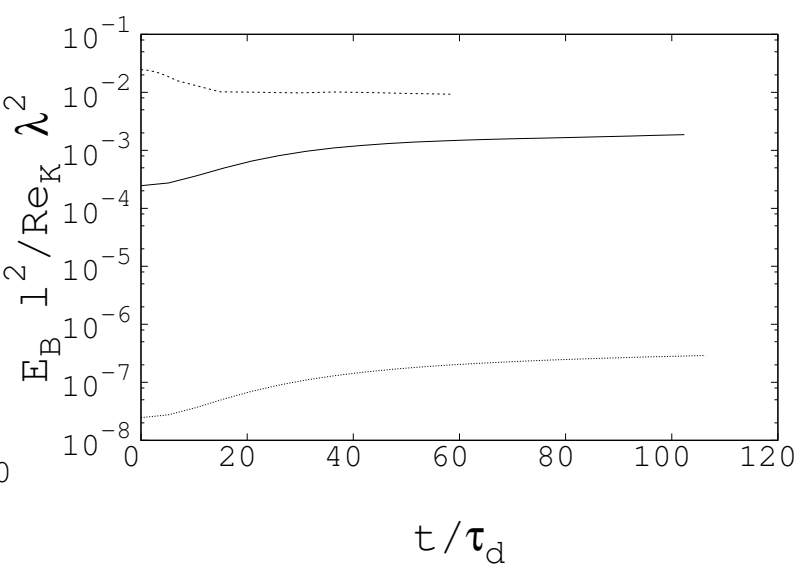

$t / \tau_{d}$

FIG. 4. $\mathrm{N}=2.5 \times 10^{-3}$. Left: Scaled $\widetilde{B}$ spectrum at $t=106.25 \tau_{\mathrm{d}}$. Right: Evolution of (scaled) $\widetilde{B}$ energy with time. From lower to upper curves, we move from low to high interaction parameter $\mathrm{N}$ values.

Fig.4 (left) shows the magnetic field $(\widetilde{B})$ spectrum $\left(t=106.25 \tau_{\mathrm{d}}\right) . \widetilde{B}$ is normalized with $\sqrt{R e_{k}} \lambda / \ell$, and distances with $\ell$ (hence the $\widetilde{B}$ spectra are normalized with $R e_{k} \lambda^{2} / \ell$, and the normalized wavenumbers are $k \ell$ ). There is a viscous cut-off close to the initial magnetic-flux ring radius (the normalization length $\ell$ ), and a maximum value at a length 


\begin{tabular}{|c|c|c|c|c|c|c|c|}
\hline Flow type & $\frac{\left\langle\lambda_{1}\right\rangle}{\left\langle S_{i j} S_{i j}\right\rangle^{1 / 2}}$ & $\frac{\left\langle\lambda_{2}\right\rangle}{\left\langle S_{i j} S_{i j}\right\rangle^{1 / 2}}$ & $\frac{\left\langle\lambda_{3}\right\rangle}{\left\langle S_{i j} S_{i j}\right\rangle^{1 / 2}}$ & $\frac{\left\langle\lambda_{1}^{2}\right\rangle}{\left\langle S_{i j} S_{i j}\right\rangle}$ & $\frac{\left\langle\lambda_{2}^{2}\right\rangle}{\left\langle S_{i j} S_{i j}\right\rangle}$ & $\frac{\left\langle\lambda_{3}^{2}\right\rangle}{\left\langle S_{i j} S_{i j}\right\rangle}$ & $\left\langle\lambda^{\star}\right\rangle$ \\
\hline MHD,$N=2.5 \times 10^{-3}$ & 0.5003 & 0.0017 & -0.5020 & 0.4852 & 0.0020 & 0.5127 & -0.0892 \\
MHD,$N=2.5 \times 10$ & 0.5019 & 0.0017 & -0.5036 & 0.4852 & 0.0020 & 0.5126 & -0.09 \\
MHD,$N=2.5 \times 10^{3}$ & 0.5071 & 0.0022 & -0.5093 & 0.4852 & 0.0029 & 0.5117 & -0.10 \\
Turbulence, $R e_{\lambda}=10^{4}$ & 0.47 & 0.06 & -0.53 & 0.41 & 0.04 & 0.55 & \\
\hline
\end{tabular}

TABLE I. Mean values of various strain-rate tensor eigenvalues related quantities for the three different values of the interaction parameter N. From small to large interaction parameter values, the corresponding times are $t=106.25 \tau_{\mathrm{d}}, t=102.38 \tau_{\mathrm{d}}, t=58.29 \tau_{\mathrm{d}}$. The corresponding values for fully developed turbulence in the atmospheric surface layer (at the height $10 \mathrm{~m}$ ) [8] are also shown for comparison.

\begin{tabular}{|c|c|c|c|c|c|}
\hline Flow type & $\frac{\left\langle\omega_{i} \omega_{j} S_{i j}\right\rangle}{\left\langle\omega_{i} \omega_{i}\right\rangle\left\langle S_{i j} S_{i j}\right\rangle^{1 / 2}}$ & $\langle\cos (\boldsymbol{\omega}, \mathbf{W})\rangle$ & $\left\langle\cos \left(\boldsymbol{\omega}, \boldsymbol{\lambda}_{1}\right)\right\rangle$ & $\left\langle\cos \left(\boldsymbol{\omega}, \boldsymbol{\lambda}_{2}\right)\right\rangle$ & $\left\langle\cos \left(\boldsymbol{\omega}, \boldsymbol{\lambda}_{3}\right)\right\rangle$ \\
\hline$N=2.5 \times 10^{-3}$ & $0.6802 \times 10^{-1}$ & $-0.5570 \times 10^{-2}$ & -0.471 & $0.237 \times 10^{-1}$ & $0.481 \times 10^{-2}$ \\
$N=2.5 \times 10$ & $0.6694 \times 10^{-1}$ & $-0.3471 \times 10^{-2}$ & -0.487 & $0.237 \times 10^{-1}$ & $0.403 \times 10^{-2}$ \\
$N=2.5 \times 10^{3}$ & $0.6166 \times 10^{-1}$ & $0.1735 \times 10^{-2}$ & -0.580 & $0.270 \times 10^{-1}$ & $-0.182 \times 10^{-2}$ \\
\hline
\end{tabular}

TABLE II. Mean values of vorticity stretching related quantities for the three different values of the interaction parameter N. From small to large interaction parameter values, the corresponding times are $t=106.25 \tau_{\mathrm{d}}, t=102.38 \tau_{\mathrm{d}}, t=58.29 \tau_{\mathrm{d}}$.

\begin{tabular}{|c|c|c|c|c|c|}
\hline Flow type & $\frac{\left\langle B_{i} B_{j} S_{i j}\right\rangle}{\left\langle B_{i} B_{i}\right\rangle\left\langle S_{i j} S_{i j}\right\rangle^{1 / 2}}$ & $\langle\cos (\mathbf{B}, \mathbf{M})\rangle$ & $\left\langle\cos \left(\mathbf{B}, \boldsymbol{\lambda}_{1}\right)\right\rangle$ & $\left\langle\cos \left(\mathbf{B}, \boldsymbol{\lambda}_{2}\right)\right\rangle$ & $\left\langle\cos \left(\mathbf{B}, \boldsymbol{\lambda}_{3}\right)\right\rangle$ \\
\hline$N=2.5 \times 10^{-3}$ & 0.02970 & 0.03092 & $0.2822 \times 10^{-2}$ & $-0.6577 \times 10^{-3}$ & $-0.2503 \times 10^{-1}$ \\
$N=2.5 \times 10$ & 0.03172 & 0.03029 & $0.3083 \times 10^{-3}$ & $-0.8622 \times 10^{-3}$ & $-0.2471 \times 10^{-1}$ \\
$N=2.5 \times 10^{3}$ & 0.05927 & 0.01781 & $-0.1263 \times 10^{-2}$ & $0.7009 \times 10^{-2}$ & $-0.1397 \times 10^{-1}$ \\
\hline
\end{tabular}

TABLE III. Mean values of magnetic-field stretching related quantities for the three different values of the interaction parameter N. From small to large interaction parameter values, the corresponding times are $t=106.25 \tau_{\mathrm{d}}, t=102.38 \tau_{\mathrm{d}}, t=58.29 \tau_{\mathrm{d}}$. 


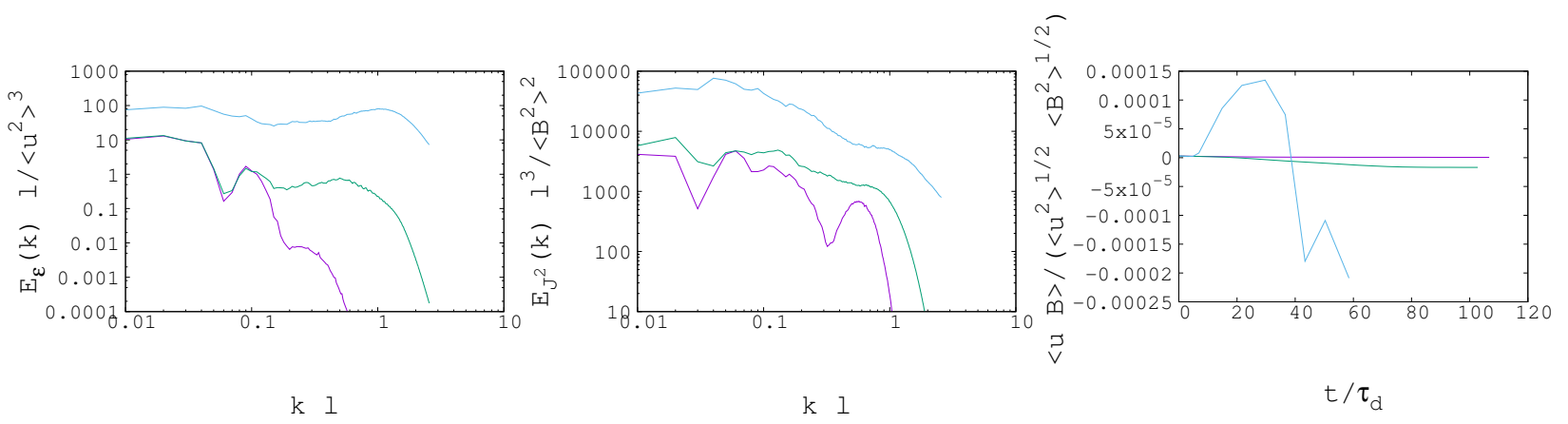

FIG. 5. Left: Scaled spectra of kinetic energy dissipation rate $\epsilon\left[\mathrm{N}=2.5 \times 10^{-3}\right.$ (lower curve), $\mathrm{N}=2.5 \times 10$ (middle curve), $\mathrm{N}=2.5 \times 10^{3}$ (upper curve)]. Middle: Scaled spectra of $\widetilde{\mathbf{J}}^{2}$ (proportional to magnetic-energy dissipation) $\left[\mathrm{N}=2.5 \times 10^{-3}\right.$ (lower curve), $\mathrm{N}=2.5 \times 10$ (middle curve), $\mathrm{N}=2.5 \times 10^{3}$ (upper curve)]. Right: Scaled cross helicity $\mathrm{H}^{\mathrm{C}}=\mathbf{u} \cdot \mathbf{B}$ versus scaled time $\left[\mathrm{N}=2.5 \times 10^{-3}\right.$ (horizontal curve), $\mathrm{N}=2.5 \times 10$ (curve close to the horizontal), $\mathrm{N}=2.5 \times 10^{3}$ (strongly varying curve)]. From small to large interaction parameter values, the corresponding times are $t=106.25 \tau_{\mathrm{d}}, t=102.38 \tau_{\mathrm{d}}, t=58.29 \tau_{\mathrm{d}}$.

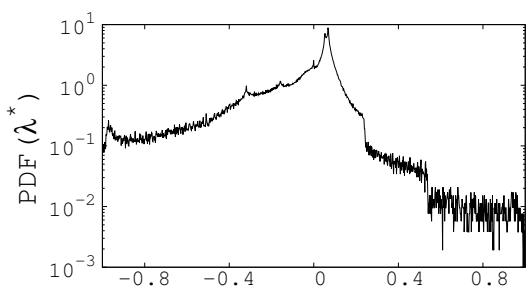

$\lambda^{*}$

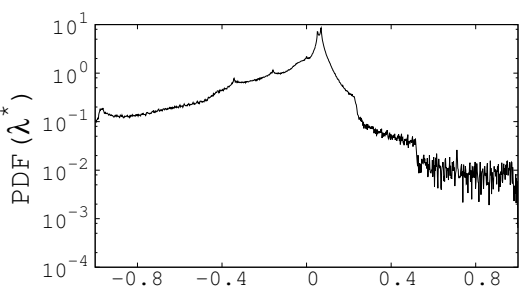

$\lambda^{*}$

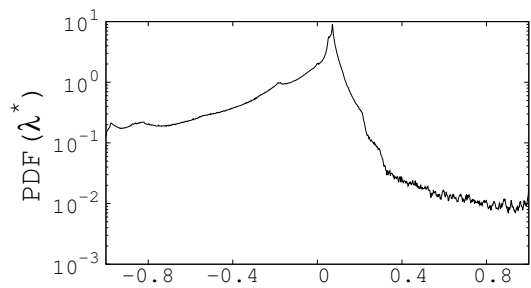

$\lambda^{\star}$

FIG. 6. Probability density functions of $\lambda^{*}$ parameter for $\mathrm{N}=2.5 \times 10^{-3}$ (left), $\mathrm{N}=2.5 \times 10$ (middle), and $\mathrm{N}=2.5 \times 10^{3}$ (right). From small to large interaction parameter values, the corresponding times are $t=106.25 \tau_{\mathrm{d}}, t=102.38 \tau_{\mathrm{d}}, t=58.29 \tau_{\mathrm{d}}$.

scale an order of magnitude larger. This "energy containing" length scale is of the order of the length of the steps of the spiral, where (as shown in Fig.3) stretched magnetic field structures are formed due to the tidal effect of the vortex flow. Since the vortex tube stretches the initial ring, it generates more magnetic field, hence, as shown in Fig.4 (right), the magnetic-field $(\widetilde{B})$ energy grows with time. Due to the small $N$ number, we do not anticipate significant "electrodynamic" vorticity generation. These are in agreement with quantitative measures of enstrophy (Table II) and magnetic-energy (Table III) sources, which indicate small amplifications of these quantities. Fig. 5 shows the kinetic (left) and magnetic 


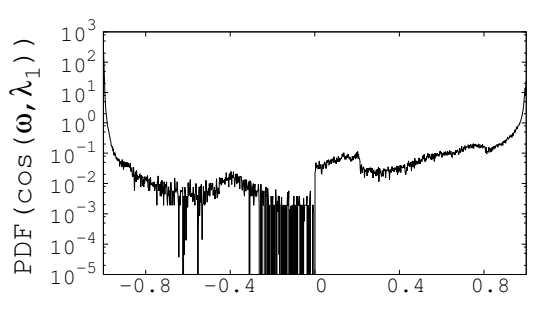

$\cos \left(\omega, \lambda_{1}\right)$

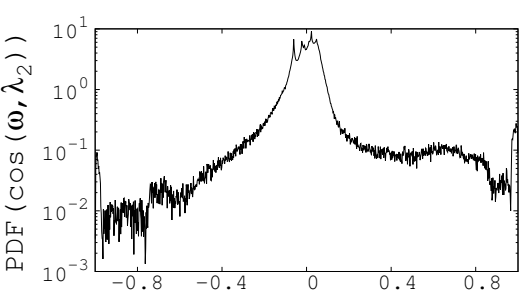

$\cos \left(\omega, \lambda_{2}\right)$

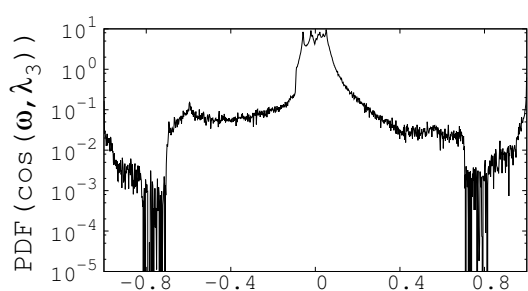

$\cos \left(\omega, \lambda_{3}\right)$

FIG. 7. Probability density functions of vorticity/strain-rate-eigenvector cosines for $N=2.5 \times$ $10^{-3}$ at time $t=106.25 \tau_{\mathrm{d}}$. From left to right: $\cos \left(\boldsymbol{\omega}, \boldsymbol{\lambda}_{1}\right), \cos \left(\boldsymbol{\omega}, \boldsymbol{\lambda}_{2}\right)$, and $\cos \left(\boldsymbol{\omega}, \boldsymbol{\lambda}_{3}\right)$.

energy (middle) dissipation rate spectra. The kinetic energy dissipation rate is computed via the expression $\epsilon=2 \nu S_{i j} S_{i j}$, where $S_{i j}=\frac{1}{2}\left(\partial u_{i} / \partial x_{j}+\partial u_{j} / \partial x_{i}\right)$ is the strain rate tensor, and the magnetic energy dissipation rate via $\mathbf{J}^{2} / \sigma$, hence, up to a scaling factor, it is proportional to the square of electric current. As expected, $\epsilon$ spectra are larger at low wavenumbers, because at small distances the fluid inside the tube rotates like a solid body, so the dissipation there is minimal. On the other hand, the magnetic dissipation ought to correspond to the electric current rings shown in Fig.3 (right), which appear over many diameter scales, so the $\mathbf{J}^{2}$ spectra are more uniformly distributed. Moreover, in agreement with the discussion of helicity invariants in ideal flow, we have checked the evolution of cross helicity $\mathrm{H}^{\mathrm{C}}$ (Fig.5). We have verified that the computation preserves the initial level of $\mathrm{H}^{\mathrm{C}}$ (which was of the order of $10^{-6}$ ), hence, there is no $\mathrm{H}^{\mathrm{C}}$ generation during the formation of the magnetic-field spiral. This is shown in Fig.5 (right) (horizontal line).

The PDF of $\lambda^{\star}$ (Fig.6) shows that (locally) the most probable flow field is similar to a planar shear flow, and, despite the fact that the PDF maximum value is shifted towards the positive $\lambda^{\star}$ axis, the mean value $\left\langle\lambda^{\star}\right\rangle$ in Table I is negative, since strongly negative $\lambda^{\star}$ values are more frequent than strongly positive values. Figures 7 and 8 show that there is small enstrophy production in the system (PDF of enstrophy production peaks at zero, but is emphatically shifted [with small probabilities] towards positive values), that is mainly due to a preferable (anti)alignment of vorticity vector with extensional eigenvector $\boldsymbol{\lambda}_{1}$, and vortex stretching vector W. The corresponding results for magnetic energy production (Figures 9 and 10) show small magnetic energy production, but the PDF of magnetic energy source is more symmetric in comparison with the vorticity case, and there is no predominant tendency of $\mathbf{B}$ and $\mathbf{M}$ (anti)alignment. These differences between vorticity and magnetic field dynamics 

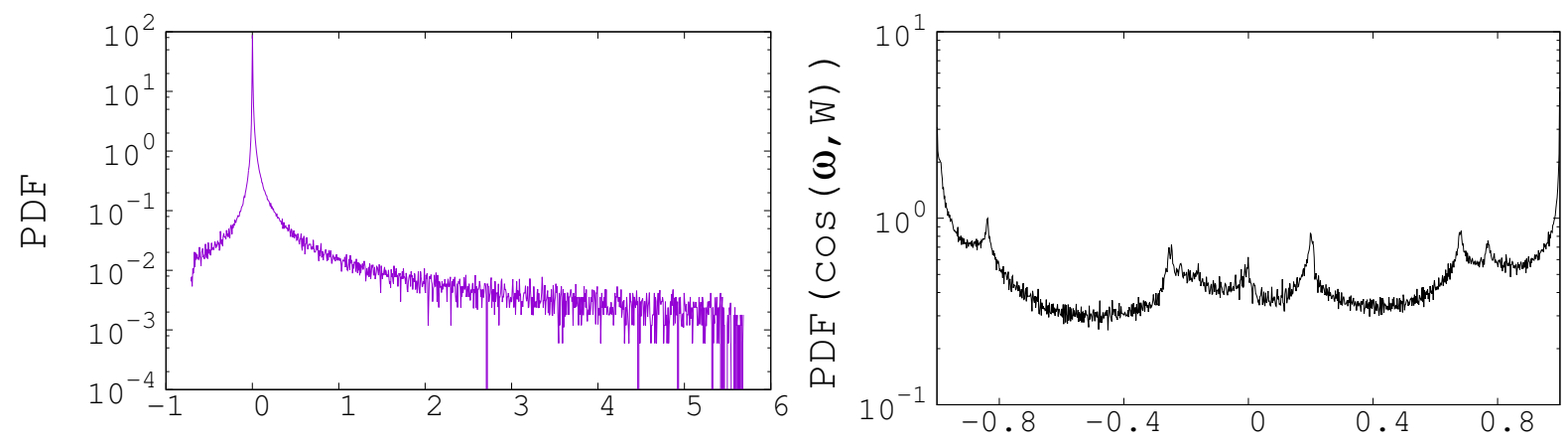

$$
\omega_{i} \quad \omega_{j} \quad S_{i j} /<\omega_{i} \omega_{i}><S_{i j} \quad S_{i j}>^{1 / 2}
$$

$\cos (\omega, W)$

FIG. 8. Left: Probability density function of scaled enstrophy source $\frac{\left\langle\omega_{i} \omega_{j} S_{i j}\right\rangle}{\left\langle\omega_{i} \omega_{i}\right\rangle\left\langle S_{i j} S_{i j}\right\rangle^{1 / 2}}$. Right: Probability density function of vorticity/vortex-stretching vector $\operatorname{cosine} \cos (\boldsymbol{\omega}, \mathbf{W})$. Both results are for $N=2.5 \times 10^{-3}$ at time $t=106.25 \tau_{\mathrm{d}}$.

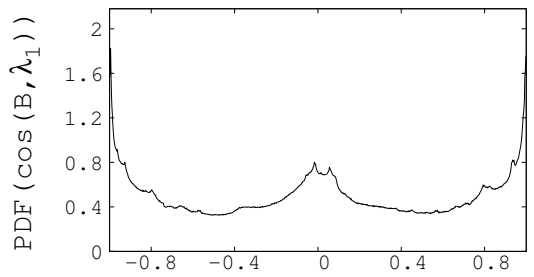

$\cos \left(B, \lambda_{1}\right)$

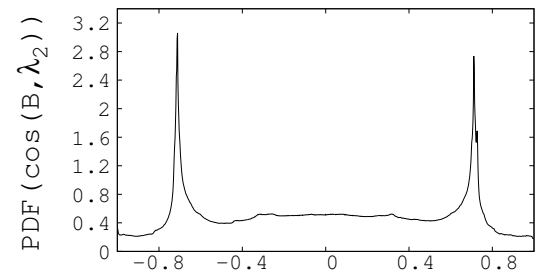

$\cos \left(B, \lambda_{2}\right)$

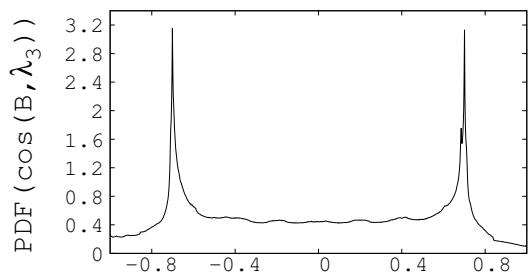

$\cos \left(B, \lambda_{3}\right)$

FIG. 9. Probability density functions of magnetic-field/strain-rate-eigenvector cosines for $N=$ $2.5 \times 10^{-3}$ at time $t=106.25 \tau_{\mathrm{d}}$. From left to right: $\cos \left(\mathbf{B}, \boldsymbol{\lambda}_{1}\right), \cos \left(\mathbf{B}, \boldsymbol{\lambda}_{2}\right)$, and $\cos \left(\mathbf{B}, \boldsymbol{\lambda}_{3}\right)$.
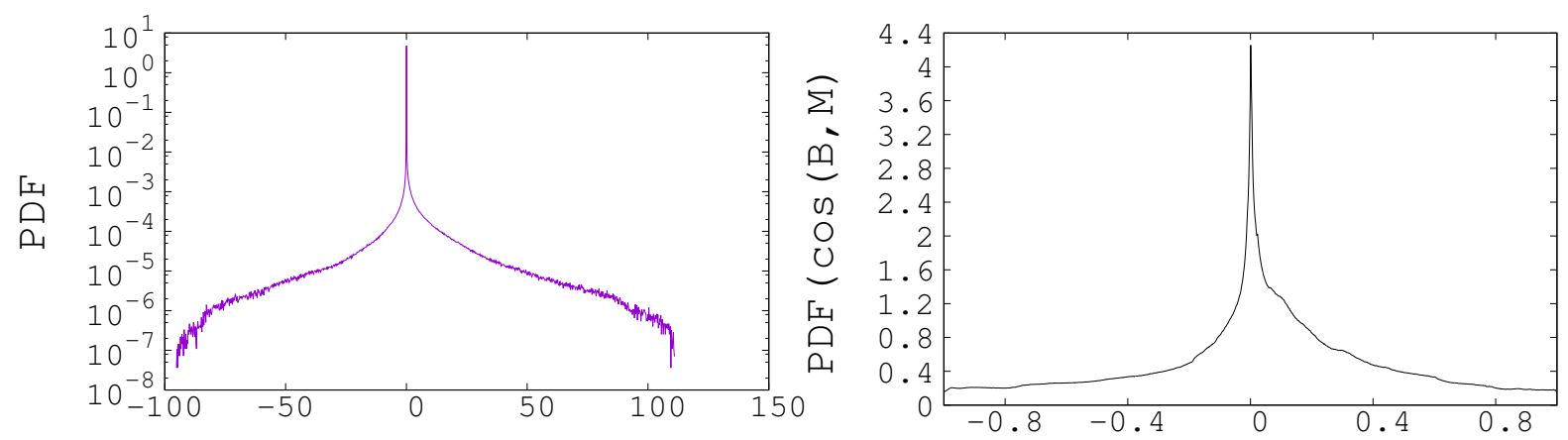

$$
B_{i} \quad B_{j} \quad S_{i j} /<B_{i} \quad B_{i}><S_{i j} S_{i j}>^{1 / 2}
$$

$\cos (B, M)$

FIG. 10. Left: Probability density function of scaled magnetic-energy source $\frac{\left\langle B_{i} B_{j} S_{i j}\right\rangle}{\left\langle B_{i} B_{i}\right\rangle\left\langle S_{i j} S_{i j}\right\rangle^{1 / 2}}$. Right: Probability density function of magnetic-field/magnetic-field stretching vector cosine $\cos (\mathbf{B}, \mathbf{M})$. Both results are for $N=2.5 \times 10^{-3}$ at time $t=106.25 \tau_{\mathrm{d}}$. 

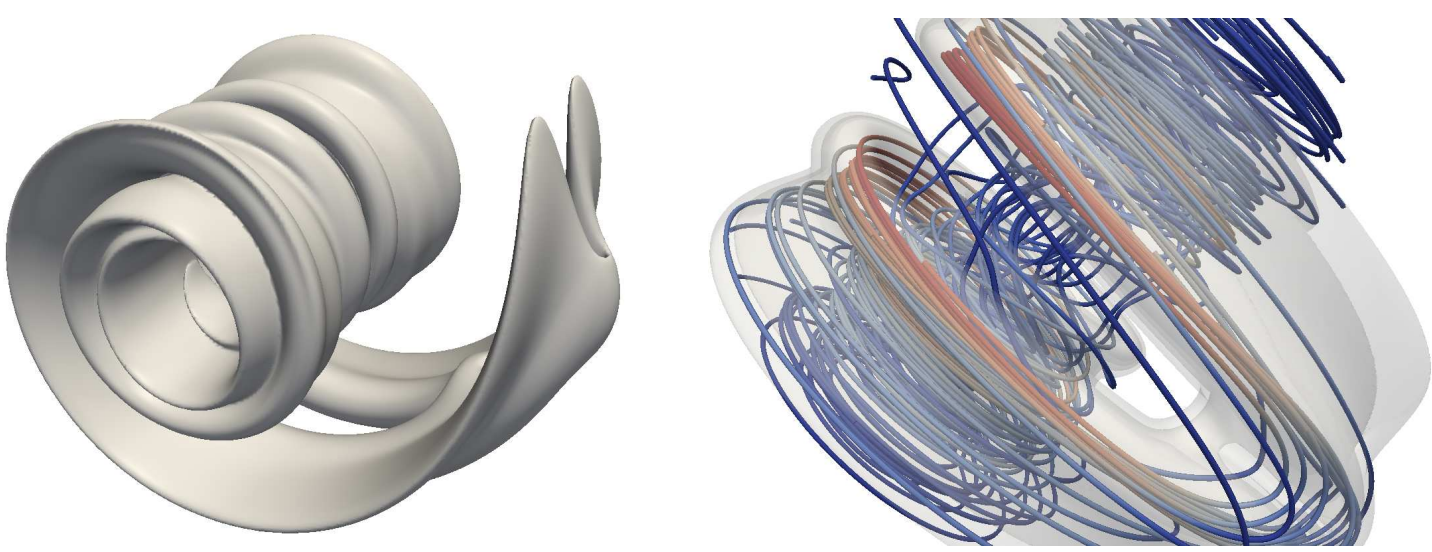

FIG. 11. $\mathrm{N}=2.5 \times 10, t=102.38 \tau_{\mathrm{d}}$. Left: Under the influence of the vortex-tube, the magneticflux ring forms a spiral. In contrast to the small interaction number case, the spiral arms are not flat but present well-formed bulges at their boundaries. Right: The magnetic field lines are smooth (without any reversals) within the spiral arms. The highest field values are attained within the bulges. In both figures, isosurfaces for ten values spanning the whole range between minimum and maximum magnetic field magnitudes are shown simultaneously. The field-lines are computed via the Runge-Kutta-Fehlberg numerical integration method.

are due to the passive-vector character of the latter in this (kinematic dynamo) flow (as also discussed in the Prologue).

COMPUTATIONAL SOLUTION FOR $R e_{k}=R e_{m}=10^{4}, N=2.5 \times 10$

Similarly with the small N case, the magnetic-flux ring evolves into a characteristic spiral structure (Fig.11, left). This is consistent with the (average) eigenvalues of the local strain-rate tensor (Table I) that are very similar with the corresponding values in the $\mathrm{N}=2.5 \times 10^{-3}$ case. Similar conclusions hold for the $\left\langle\lambda^{\star}\right\rangle$ value in Table I, and the probability density function of $\lambda^{\star}$ (Fig.6, middle). However, an important difference here is the formation of characteristic bulges at spiral arms boundaries. The magnetic field attains its highest values within these bulges (Fig.11, right). The new, curved shape of the flattened spiral-arms surfaces ought to be related to Lorentz force action, and vorticity generation. Indeed, Fig.12 (left) shows the Lorentz force generated, high-intensity vorticity regions at the magnetic-field bulges. This effect is due to the higher (as compared with the kinematic case) interaction parameter value, that allows the Lorentz force to significantly impact on 

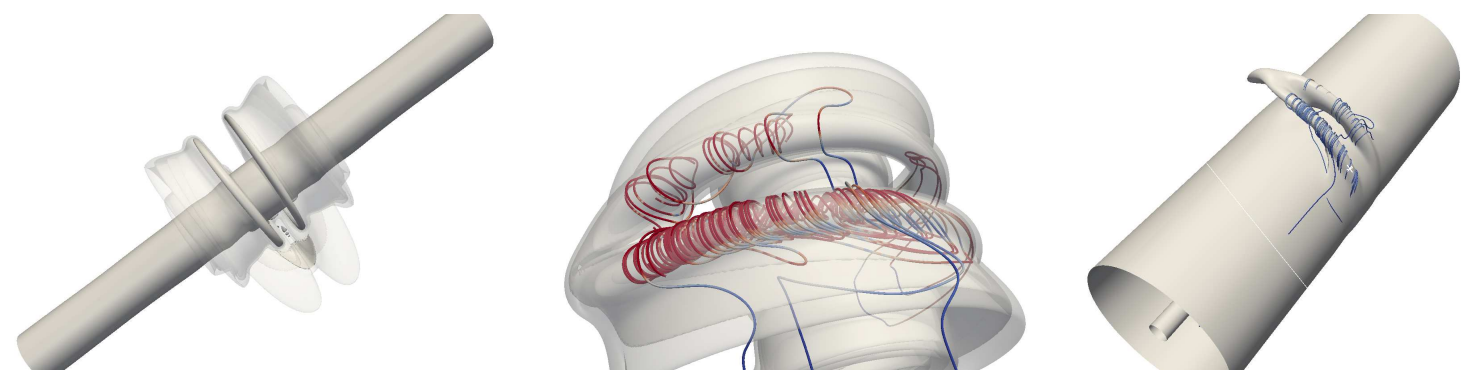

FIG. 12. $\mathrm{N}=2.5 \times 10, t=102.38 \tau_{\mathrm{d}}$. Left: Magnetic-field isosurfaces (low opacity) shown together with vorticity isosurfaces. The highest vorticity values are located at the magnetic-field bulges. The vortex tube presents two indentations at the areas where the magnetic spiral is closest to it. Middle: Intriguing solenoid-like structure of vorticity-field lines (colored field-lines) located at the magnetic field bulges (background isosurface). It indicates strong Lorentz force effects. The color code refers to the vorticity-field magnitude, with the blue color corresponding to the smaller, and the red color to the larger values. Not all vortex lines within the solenoid have equally large vorticity magnitudes with those shown here. Right: Velocity isosurface at level equal to 0.5 of maximum value. The large (cylindrical) isosurface corresponds to the (undisturbed) potential vortex-tube induced flow. The smaller inner cylinder is within the tube's solid body rotation region. The superposed vortex-line solenoid helps to indicate the Lorentz-force induced vortexstructure with increased (relatively to the undisturbed vortex flow) velocity there. The field-lines are computed via the Runge-Kutta-Fehlberg numerical integration method.

inertial flow processes. Fig.12 (middle) indicates that the vorticity structure generated by the Lorentz force is a solenoid. Hence, according to the results, the Lorentz force accelerates the flow within this solenoid, whilst generating a "counterflow" (with respect to the undisturbed vortex flow) in the adjacent fluid within the magnetic spiral. It is important to note here that, although the vorticity-solenoid is consistent with a jet flow within the coils, the induced-velocity magnitudes are not large enough to create a distinct jet flow structure, and the flow (outside the initial vortex-tube core) remains a mildly deformed potential vortex flow. This is explicit in Fig.12 (right), where a velocity isosurface is shown. The large cylindrical surface corresponds to the undisturbed potential flow field at this distance from the vortex axis, and the inner cylinder to the solid body rotation within the vortex (since the potential flow outside the vortex is decreasing with distance, there is always a match between a velocity level within the vortex-core, and the velocity at some distance from the vortex 

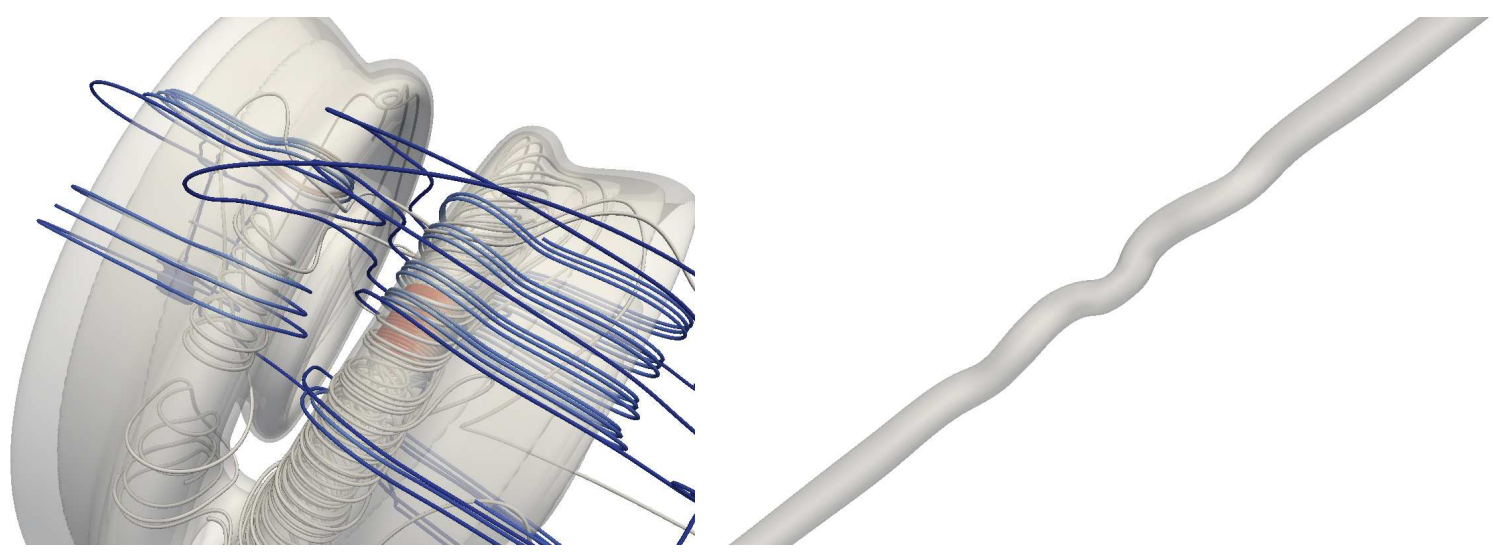

FIG. 13. $\mathrm{N}=2.5 \times 10, t=102.38 \tau_{\mathrm{d}}$. Left: Similarly with the small interaction number case, the current-lines (colored tubes) engulf the arms of the magnetic spiral. However, at places, they acquire a solenoid like structure within the vorticity solenoid structure (white tubes). The current solenoids are associated with the highest current values. Right: Isosurface of vorticity (at a level equal to 0.83 of maximum value) located within the initial tube area. Due to Lorentz force action, the magnetic spiral acts like a vortex-tube constrictor, deforming the (initially cylindrical) isosurface. The field-lines are computed via the Runge-Kutta-Fehlberg numerical integration method.

axis). We observe that the velocity within the vorticity-solenoid is equal to the velocity at a smaller distance from the vortex (i.e., equal to a higher value than the one corresponding to the undisturbed potential flow velocity at the locations of the structure). Moreover, as shown in Fig.13 (left), and in agreement with the small $\mathrm{N}$ case, weak-current lines tend to engulf the magnetic-field spiral arms, whilst closed current loops of higher intensity are formed within the magnetic structure. An intriguing feature here is that the closed loops are organized (at places) into current-solenoids within the vorticity-solenoid. These small scale currents are responsible for enlarged, high k magnetic dissipation (Fig.5, middle) as compared with the smaller interaction parameter case. On the other hand, the small scale vorticity solenoids could be responsible for the increased kinetic energy dissipation that is observed at high k (Fig.5, left). This is direct evidence of (a relatively weak) departure from kinematic dynamo picture, and is also indicated by the cross helicity results of Fig.5 (right), where a small departure from the horizontal line of the kinematic dynamo case is observed, consistent with a very weak anti-alignment between velocity and magnetic fields. Overall, the observed magnetic-field bulges are a higher $\mathrm{N}$ effect, that, however, does not alter the 


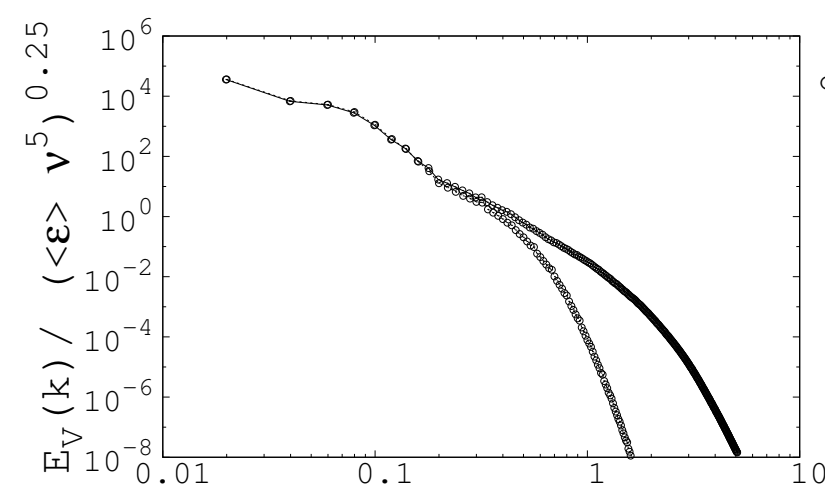

$\mathrm{k} 1$

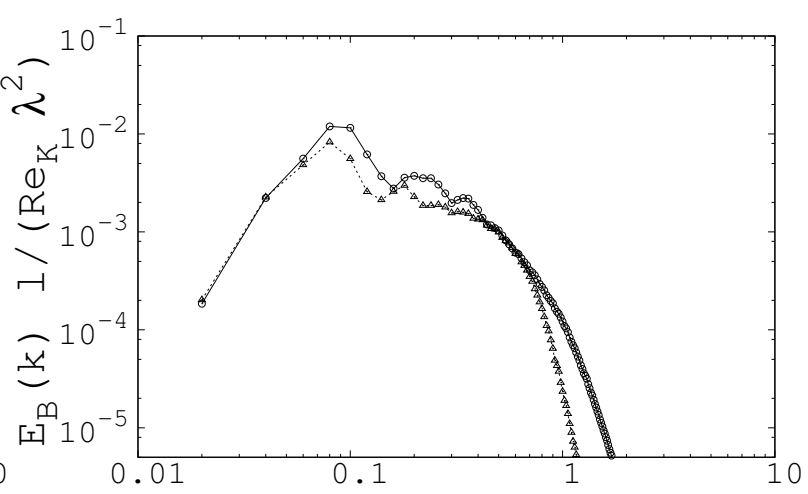

$\mathrm{k} \quad 1$

FIG. 14. $\mathrm{N}=2.5 \times 10, t=102.38 \tau_{\mathrm{d}}$. Left: Kinetic energy spectra for $N=2.5 \times 10^{-3}$ (faster cut-off), and $N=2.5 \times 10$. In latter case, there is evidence of a high wavenumber $k^{-4}$ scaling regime induced by the magnetic-field's reaction to the flow, which follows a steeper $k^{-5}$ regime that appears in both spectra, and corresponds to the tube core. Right: Normalized magnetic energy spectra. The $N=2.5 \times 10^{-3}$ results (triangles) have been multiplied by a large factor in order to compare them with the $N=2.5 \times 10$ results (circles).

grand picture of spiral wrapping of the field around the vortex. Another important higher $\mathrm{N}$ effect (Fig.13, right) has to do with the fact that, the magnetic-field spiral is not passive, but, like a constrictor, deforms (an initially cylindrical) high vorticity isosurface within the vortex-tube volume.

As shown in Fig.4 (right), the magnetic field energy increases with time. Hence, despite the fact that some of the magnetic field energy is transferred to the fluid (in order to generate the vorticity-solenoid structure), on the average, the transfer of energy is from the fluid to the field. Due to nontrivial Lorentz force effects, it is helpful to compute the kinetic-energy spectra. Indeed, Fig.14 (left) superposes the (normalized) kinetic spectra for the $N=2.5 \times 10^{-3}$, and $N=2.5 \times 10$ cases. The spectrum is normalized with the factor $\left(\langle\epsilon\rangle \nu^{5}\right)^{1 / 4}$, where $\langle\epsilon\rangle$ is the volume average of the kinetic energy dissipation rate $\epsilon=2 \nu S_{i j} S_{i j}$. The key difference of the two cases is the appearance of a high wavenumber, $k^{-4}$ scaling regime in the larger $\mathrm{N}$ case, that pushes the dissipative exponential cut-off towards higher $k$. Since, this effect can only be associated with the Lorentz force action discussed previously, we can conclude that this spectral regime corresponds to the induced solenoid-like vorticity regions at the magnetic-spiral edges. This high $k$ regime could be observed (for example) in turbulent dy- 


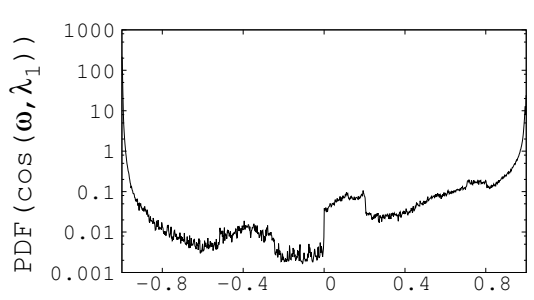

$\cos \left(\omega, \lambda_{1}\right)$

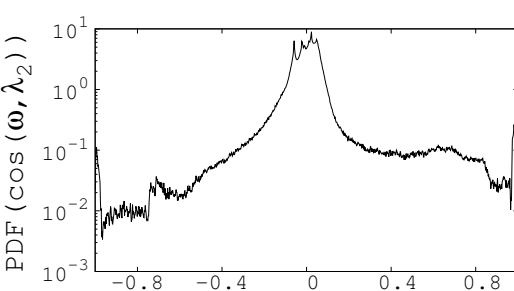

$\cos \left(\omega, \lambda_{2}\right)$

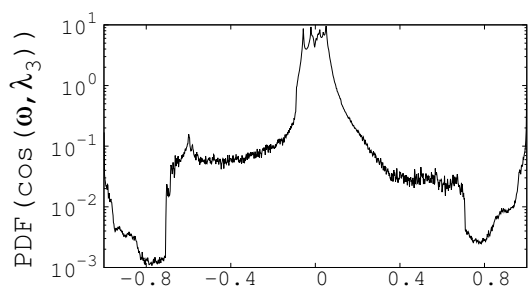

$\cos \left(\omega, \lambda_{3}\right)$

FIG. 15. Probability density functions of vorticity/strain-rate-eigenvector cosines for $N=2.5 \times 10$ at time $t=102.38 \tau_{\mathrm{d}}$. From left to right: $\cos \left(\boldsymbol{\omega}, \boldsymbol{\lambda}_{1}\right), \cos \left(\boldsymbol{\omega}, \boldsymbol{\lambda}_{2}\right)$, and $\cos \left(\boldsymbol{\omega}, \boldsymbol{\lambda}_{3}\right)$.
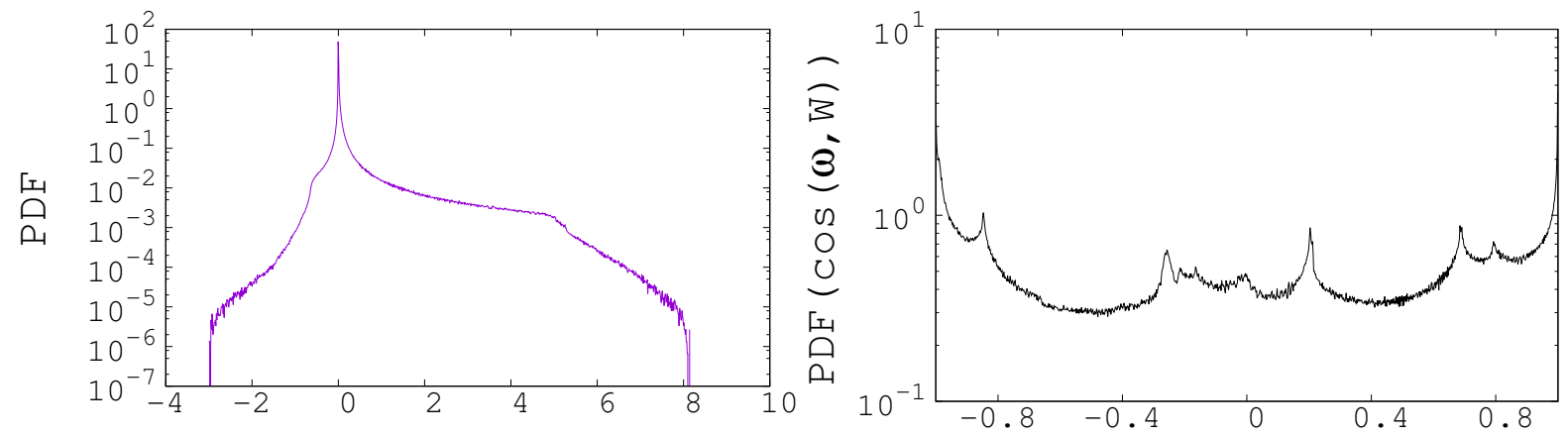

$$
\omega_{i} \omega_{j} \quad S_{i j} /<\omega_{i} \quad \omega_{i}><S_{i j} S_{i j}>^{1 / 2}
$$

$\cos (\omega, w)$

FIG. 16. Left: Probability density function of scaled enstrophy source $\frac{\left\langle\omega_{i} \omega_{j} S_{i j}\right\rangle}{\left\langle\omega_{i} \omega_{i}\right\rangle\left\langle S_{i j} S_{i j}\right\rangle^{1 / 2}}$. Right: Probability density function of vorticity/vortex-stretching vector $\operatorname{cosine} \cos (\boldsymbol{\omega}, \mathbf{W})$. Both results are for $N=2.5 \times 10$ at time $t=102.38 \tau_{\mathrm{d}}$.

namo computations, signaling the end of the kinematic dynamo regime [21], and the start of reciprocal field-fluid interactions. Notably, around $k \ell \approx 0.15$, there is a steeper $k^{-5}$ spectral slope that corresponds to the vortex tube core, and was analytically computed in [43]. The shape of the magnetic field spectra (Fig.14, right) compares well with the corresponding shape of $N=2.5 \times 10^{-3}$ spectra. Both spectra show a similar low wavenumber $k^{3}$ slope, but the $N=2.5 \times 10$ spectrum presents (relatively) higher energy levels, in the region following the peak of the spectrum.

Probing the dynamics (Figures 15-16), we see that the production of enstrophy recorded in Table I is associated with the tendency of vorticity to (anti)align with the extensional eigenvector $\boldsymbol{\lambda}_{1}$ and the vortex stretching vector $\mathbf{W}$ (the other two eigenvectors tend to be normal to the vorticity). Although, in a similar fashion with the $N=2.5 \times 10^{-3}$ case, the probablity 


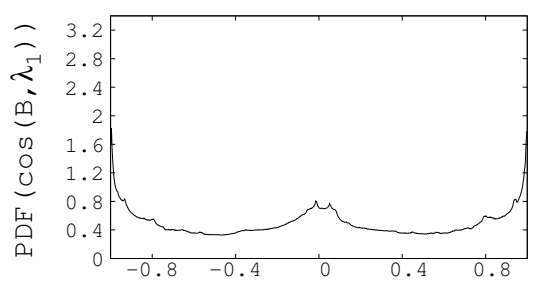

$\cos \left(B, \lambda_{1}\right)$

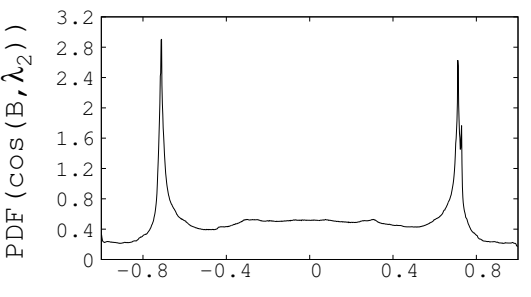

$\cos \left(B, \lambda_{2}\right)$

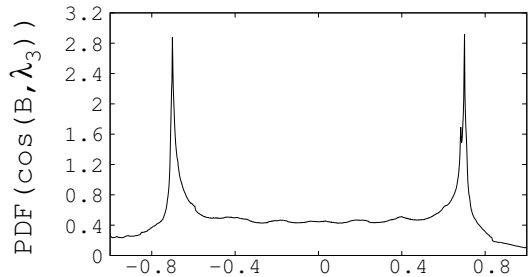

$\cos \left(B, \lambda_{3}\right)$

FIG. 17. Probability density functions of magnetic-field/strain-rate-eigenvector cosines for $N=$ $2.5 \times 10$ at time $t=102.38 \tau_{\mathrm{d}}$. From left to right: $\cos \left(\mathbf{B}, \boldsymbol{\lambda}_{1}\right), \cos \left(\mathbf{B}, \boldsymbol{\lambda}_{2}\right)$, and $\cos \left(\mathbf{B}, \boldsymbol{\lambda}_{3}\right)$.
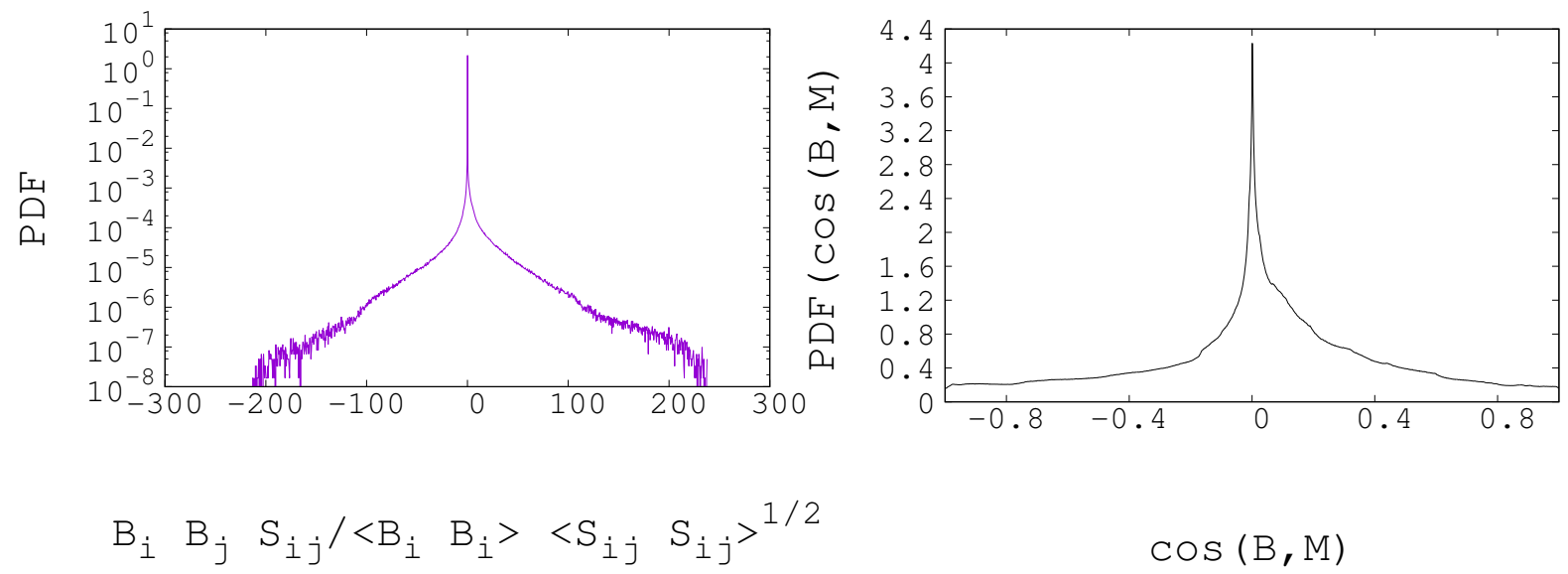

FIG. 18. Left: Probability density function of scaled magnetic-energy source $\frac{\left\langle B_{i} B_{j} S_{i j}\right\rangle}{\left\langle B_{i} B_{i}\right\rangle\left\langle S_{i j} S_{i j}\right\rangle^{1 / 2}}$. Right: Probability density function of magnetic-field/magnetic-field stretching vector cosine $\cos (\mathbf{B}, \mathbf{M})$. Both results are for $N=2.5 \times 10$ at time $t=102.38 \tau_{\mathrm{d}}$.

density function of enstrophy production peaks at zero, a new element here is the appearance of more extreme values, albeit, with very small probabilities. The magnetic field dynamics indicate (Fig.17) that all strain-rate eigenvectors (and not just the extensional eigenvector) show strong (anti)alignments with B. On the other hand, although the probability density function of the magnetic energy source (Fig.18), (similarly with the $N=2.5 \times 10^{-3}$ case) peaks at zero value, it now extends over a larger range of values, with strong amplification events slightly more intense than strong reduction events. 

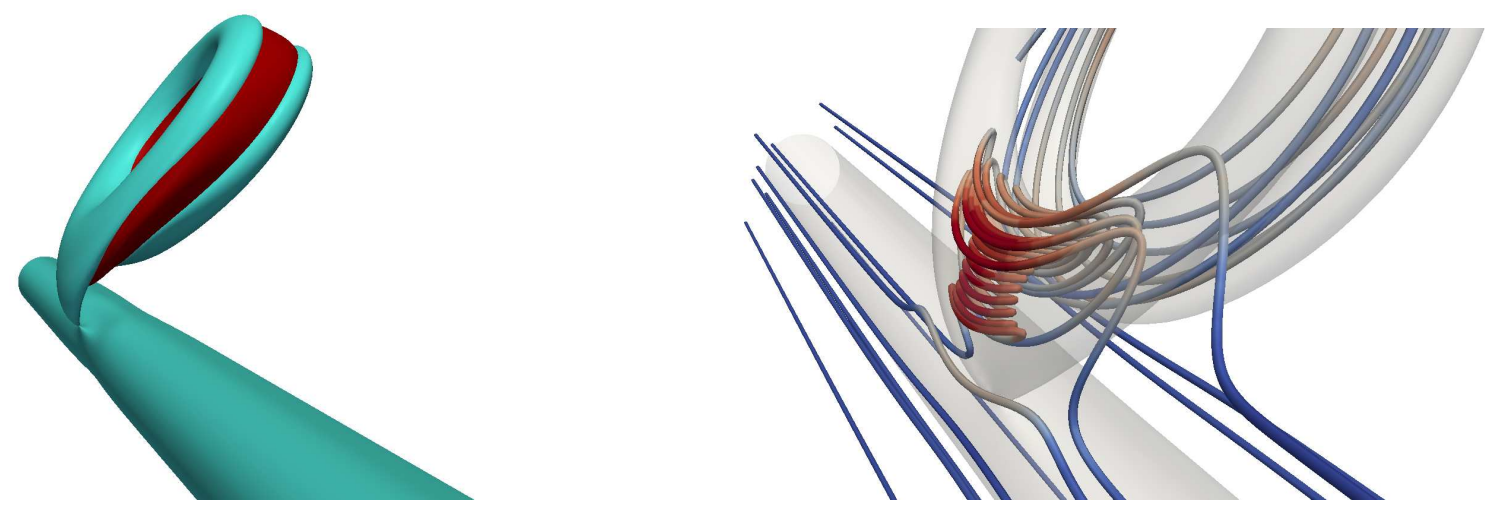

FIG. 19. $\mathrm{N}=2.5 \times 10^{3}, t=5.07 \tau_{\mathrm{d}}$. Left: The electrodynamic vorticity source creates two vortex rings that "sandwich" the initial magnetic ring (middle structure). One of the newly created vortex rings reconnects with the tube. Right: Although the vortex lines within the vortex rings are smooth and circular, the ring-tube reconnection region is characterized by highly coiled vortex lines. Such a coiled vortex-line has reconnected with two vortex lines, that (in the undisturbed flow field) ran parallel to the tube. The vorticity isosurface is drawn at level equal to 0.32 of the maximum value, and the magnetic-field isosurface at level equal to 0.58 of the maximum value. The field-lines are computed via the Runge-Kutta-Fehlberg numerical integration method.

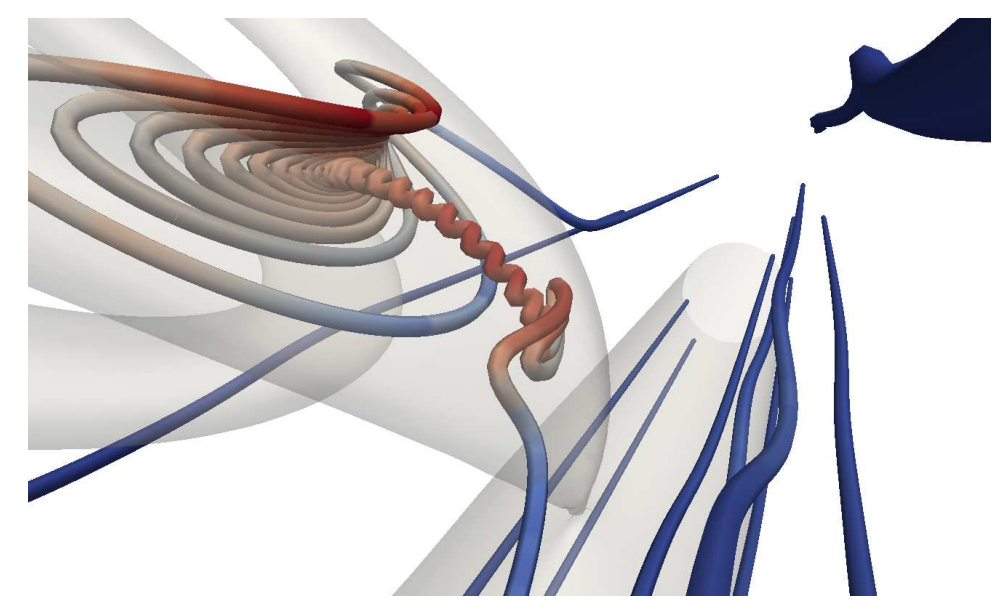

FIG. 20. $\mathrm{N}=2.5 \times 10^{3}, t=5.07 \tau_{\mathrm{d}}$. The serpentine reconnection: a vortex line belonging to a Lorentz-force induced ring in Fig.19, approaches the tube-ring contact point in a way reminiscent of a serpentine. The vorticity magnitudes within the serpentine are orders of magnitude higher than the corresponding ones within the tube. The vorticity isosurface is drawn at level equal to 0.32 of the maximum value. The field-lines are computed via the Runge-Kutta-Fehlberg numerical integration method. 
COMPUTATIONAL SOLUTION FOR $R e_{k}=R e_{m}=10^{4}, N=2.5 \times 10^{3}$

In this case (Fig.4, right), the magnetic energy of the ring is larger than the kinetic energy of the tube. It is easy to think of ways for producing similar conditions in a turbulent flow. Indeed, the latter is an hierachy of eddies/vortices that populate its inertial range. Larger eddies are characterized by larger energy, hence their circulation and Reynolds number is higher than that of the smaller eddies. Hence, it is plausible that a magnetic ring of high magnetic energy and large radius is initially formed via large-eddy dynamo action, and that it subsequently creates smaller rings via reconnections with itself or other rings. One of these highly energetic, smaller rings could interact with the smaller turbulence eddies according to our model-calculation here.

The magnetic field energy dynamics of Fig.4 (right) show that (as expected by the high N value), the Lorentz force transfers electrodynamic energy to the flow. Fig.19 (left) shows that this transfer generates (via the electrodynamic source in the vorticity equation) two vortex-rings that "sandwich" the magnetic ring. The vorticity magnitude in these rings is orders of magnitude higher than the corresponding one in the vortex tube. As usually the case in vortex dynamics, one of the rings reconnects with the vortex tube. Figures 19 (right) and 20, indicate an intriguing vortex structure in the neighborhood of this reconnection event. Indeed, although the vortex lines within the rings are circular and follow the ring perimeter, they coil and turn about each other in their route towards the point of contact with the tube. The coiling is particularly fierce for those lines that travel all the way to the contact point, presenting a serpentine-like structure (Fig.20).

High $\mathrm{N}$ physics are dominated by the effects of the Lorentz force on the flow. Fig.21 (left) shows that the initial magnetic ring has now evolved into a sheet that tends to wrap around the tube, but two most prominent magnetic rings have now developed on both of its sides. Remarkably, these two rings are not similar to the bulges we saw formed in the intermediate $\mathrm{N}$ case. The latter were formed on the ring edges along the parallel to the vortex tube direction (endowing also the flattened spiral surfaces with curvature), whilst the width of the protruding structures of Fig.21 grows along the normal (to the initial magnetic ring plane) direction. Thus, they are different structures altogether. Their origin can be explained with help of Fig.11 (right), where a rare instance of two vortex rings, a vortex sheet, and a vortex tube interwoven together is depicted. The sheet is collocated with the 

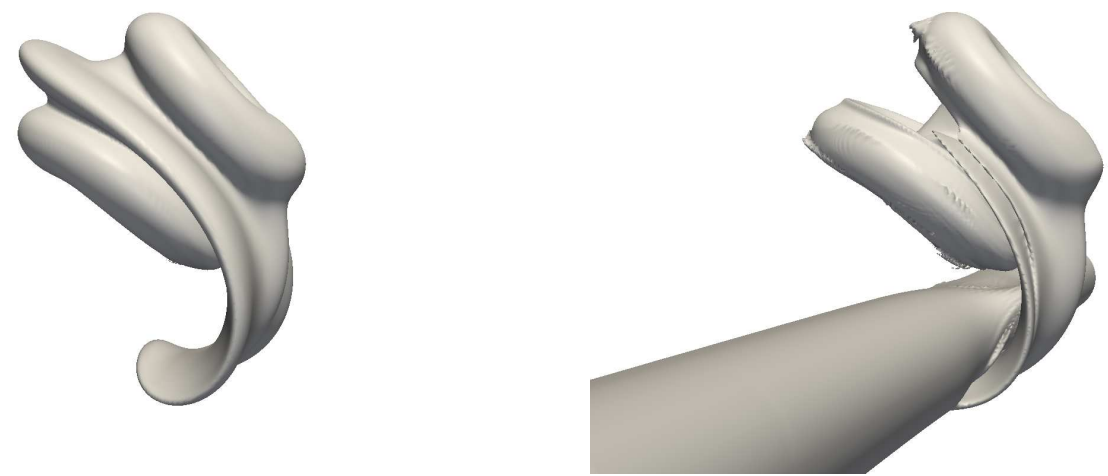

FIG. 21. $\mathrm{N}=2.5 \times 10^{3}, t=13.52 \tau_{\mathrm{d}}$. Left: Magnetic field structure: the initial ring is located at the centre of the structure having evolved into a sheet that is drawn towards the vortex tube. Two new magnetic rings are collocated with the two new vortex rings created by the Lorentz force. Right: Vorticity field structure: A rare example where, a vortex sheet, two vortex rings, and a (deformed) vortex tube are interwoven together. The vortex-sheet is collocated with the magnetic sheet in the left graphic. The two vortex rings are created by the Lorentz-force, and are also collocated with the two magnetic-rings in the left graphic. The maximum vorticity value within the induced vortex rings is 3.35 times larger than the highest vorticity value within the initial vortex tube. In both figures, isosurfaces for ten values spanning the whole range between minimum and maximum magnetic-field (left) and vorticity-field (right) magnitudes are shown simultaneously.

flattened initial magnetic ring, and is generated by Lorentz force action there. Similarly, since for smaller $\mathrm{N}$ values there was no vortex-ring generation, the two observed vortex rings (also depicted in Fig.19, left) ought to be created by Lorentz force action (i.e., the $\nabla \times(\mathbf{J} \times \mathbf{B})$ term in the vorticity equation) at the (initial) magnetic ring faces. Subsequently, the $(\mathbf{B} \cdot \nabla) \mathbf{u}$ term in the $\mathbf{B}$ equation, where $\mathbf{u}$ is now the flow field corresponding to the two vortex rings, ought to have created the two new magnetic rings in Fig.21 (left). This is a good example of nonlinear MHD effects. Tables II and III quantify these effects from various points of view: the vortex stretching vector tends to align (on average) with the direction of vorticity, whilst in the smaller interaction parameter cases, the opposite effect was observed. Similarly, the role of the the third compressive eigenvector is now different, since (on average) it tends to anti-align with the direction of vorticity. On the other hand, the (average and scaled) magnetic energy production is almost twice as large in the 
$N=2.5 \times 10^{3}$ case, a reflection of the generation of the two new magnetic-rings (Fig.21). Moreover, the probability density function of $\lambda^{\star}$ favours slightly more negative $\lambda^{\star}$ values. The generation of strongly dissipative structures leads to significant (with respect to smaller interaction parameter values) cross-helicity dynamics (Fig.5, right), as well as, significantly higher kinetic and magnetic energy dissipation-rate levels. Indeed, Fig.5 (left) shows that the low wavenumber kinetic energy dissipation-rate spectrum attains a significantly higher level than the corresponding spectrum of a straight vortex tube, whilst at high wavenumbers, a peak, that most probably corresponds to the vortex-sheet/vortex-tube reconnection process (Fig.21, right), is observed. On the other hand, the two new magnetic-rings radii (Fig.21, left) correspond to small wavenumbers, and the magnetic-energy dissipation rate also peaks at low $\mathrm{k}$. It is important to remark here the difference between small and large $\mathrm{N}$ phenomenologies: for $\mathrm{N} \ll 1$, Lorentz-force effects are negligible, and the ring behaves like a passive-vector of similar shape; for $\mathrm{N} \gg 1$ however, the key phenomenology of vortex and magnetic structure emergence is initiated by Lorentz-force induced enstrophy generation at the initial magnetic ring location, that would have occurred even in the absence of the vortex tube. By comparison, without the presence of a vortex-tube (i.e., in a uniform flow), an $\mathrm{N} \ll 1$ magnetic ring would simply grow due to diffusive action. At later times, the flow and magnetic field phenomenologies become complicated, since the self-propelled vortex rings advect also the magnetic field, hence the interactions of the latter with the vortex tube are not the dominant effects. Overall, due to the high $\mathrm{N}$ physics demonstrated above, there is a very good correlation between magnetic and vortex structures in this case. Fig.22 shows kinetic and magnetic spectra. The former present a high wavenumber $k^{-5 / 2}$ scaling regime that reflects the complicated vorticity structure discussed above. The magnetic spectra have a different shape than the spectra corresponding to small and intermediate N. In the high $\mathrm{N}$ spectra case, the energy peak is followed directly by a dissipative cut-off. The computed filling-up of the high $k$ magnetic scales is the culprit of Lorentz force action, and ought to be associated with newly generated magnetic field structures. It is helpful to note here, that in the final-time energy spectrum, the viscous cut-off range is not as extensive as in the smaller interaction parameter value cases. This does not indicate a resolution flaw, since following the evolution of the spectra from $t=0$ to the final time, we observe that, as the system evolves, more and more of the initial viscous cut-off regime is taken over by the new, Lorentz force induced scaling, but the computation stops before the available viscous cut-off 


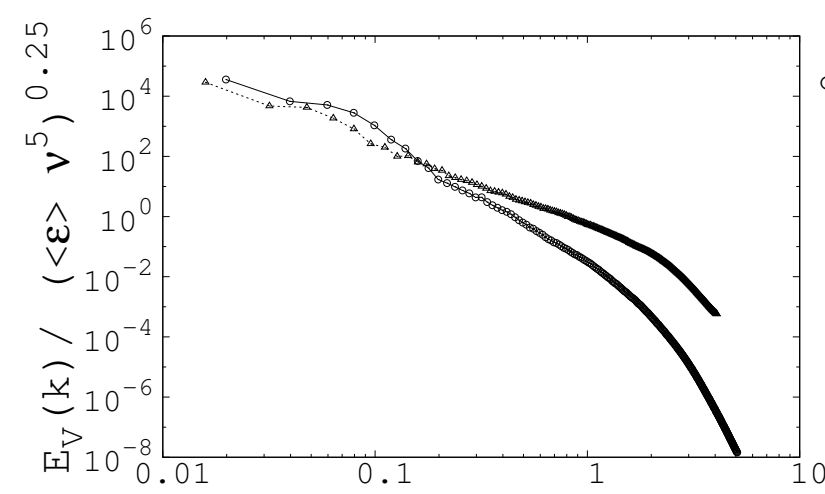

$\mathrm{k} \quad 1$

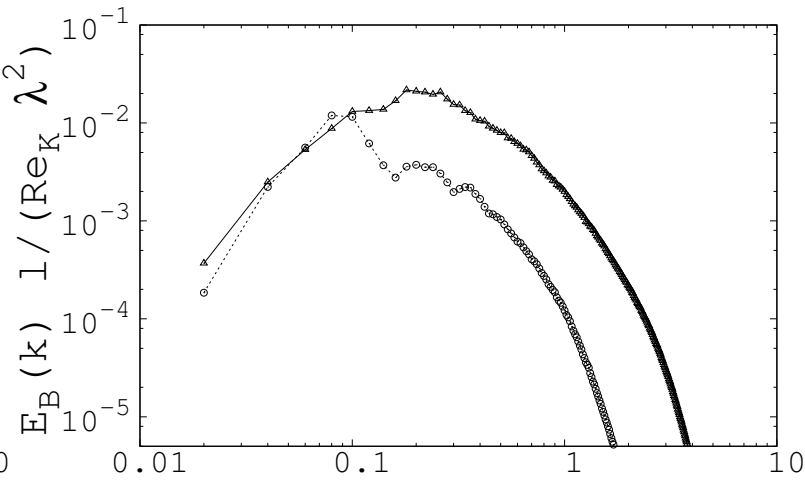

$\mathrm{k} \quad 1$

FIG. 22. $\mathrm{N}=2.5 \times 10^{3}, t=58.29 \tau_{\mathrm{d}}$. Left: Normalized kinetic energy spectra for $N=2.5 \times 10$ (circles), and $N=2.5 \times 10^{3}$ (triangles). In latter case, there is evidence of a high wavenumber $k^{-5 / 2}$ scaling regime. Right: Normalized magnetic energy spectra. The $N=2.5 \times 10^{3}$ results (upper curve, triangles) differ from the $N=2.5 \times 10$ results (circles), in that the plateau region after the peak in the latter, has now evolved into a new (displaced) peak, directly followed by dissipative cut-off.

regime is exhausted. Due to the high Reynolds number, the flow field would (at later times) develop instabilities and invalidate the numerical resolution, but this regime is not within the scope of the current investigation, not least because the vortex and magnetic structures would then become unstable, and structural coherence would be lost. Hence, throughout the high interaction parameter evolution, the resolution requirement is monitored and always satisfied, and the computation stops before the physical resolution requirements exceed the available numerical resolution. Based on these, it can be conjectured that, at much higher values of the interaction parameter, the same sequence of events (i.e., Lorentz-force induced formation of two vortex rings followed by the creation of two magnetic rings adjacent to the initial one) would be observed, but (due to the more vigorous forcing) the flow would become unstable, and transition to turbulence much faster.

When it comes to dynamical mechanisms of enstrophy and magnetic energy production, it is interesting to observe in Figures 23-26 that the related probability density functions of the alignment cosines are remarkably similar with the smaller interaction parameter cases. Certainly, this is associated with the predominant sheet-like magnetic and vortical structures in Fig.21. However, there is a key observation: both probability density functions 


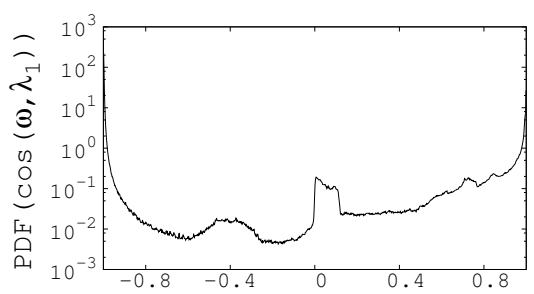

$\cos \left(\omega, \lambda_{1}\right)$

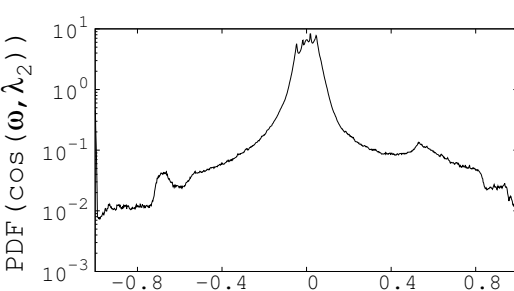

$\cos \left(\omega, \lambda_{2}\right)$

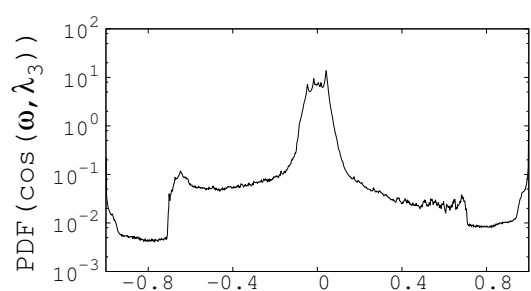

$\cos \left(\omega, \lambda_{3}\right)$

FIG. 23. Probability density functions of vorticity/strain-rate-eigenvector cosines for $N=2.5 \times 10^{3}$ at time $t=58.29 \tau_{\mathrm{d}}$. From left to right: $\cos \left(\boldsymbol{\omega}, \boldsymbol{\lambda}_{1}\right), \cos \left(\boldsymbol{\omega}, \boldsymbol{\lambda}_{2}\right)$, and $\cos \left(\boldsymbol{\omega}, \boldsymbol{\lambda}_{3}\right)$.
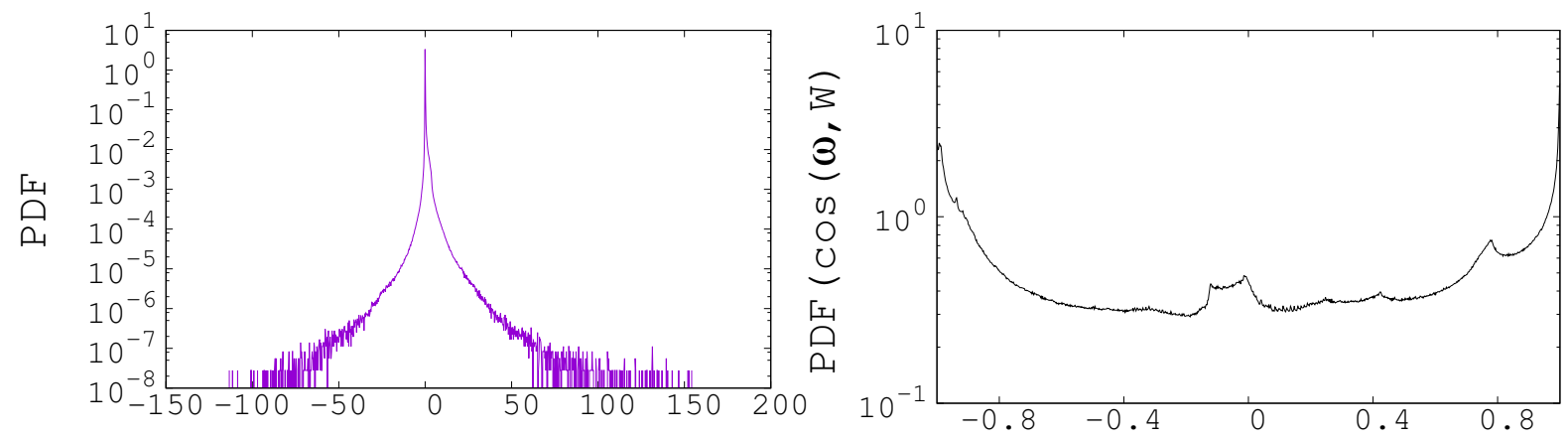

$$
\omega_{i} \quad \omega_{j} S_{i j} /<\omega_{i} \quad \omega_{i}><S_{i j} S_{i j}>^{1 / 2}
$$

$\cos (\omega, W)$

FIG. 24. Left: Probability density function of scaled enstrophy source $\frac{\left\langle\omega_{i} \omega_{j} S_{i j}\right\rangle}{\left\langle\omega_{i} \omega_{i}\right\rangle\left\langle S_{i j} S_{i j}\right\rangle^{1 / 2}}$. Right: Probability density function of vorticity/vortex-stretching vector $\operatorname{cosine} \cos (\boldsymbol{\omega}, \mathbf{W})$. Both results are for $N=2.5 \times 10^{3}$ at time $t=58.29 \tau_{\mathrm{d}}$.

for enstrophy and magnetic-energy production peak at zero, and present long tails towards much larger values than in the lower interaction parameter cases. Hence, it follows the important conclusion, that the net amplifications of enstrophy and magnetic-energy are due to the slight imbalance between extreme amplification and reduction events, i.e., they are "long tails" effects.

\section{EPILOGUE}

From a certain point of view, fluid turbulence is made possible via the interaction between inertial and pressure fields. The vortex structure of turbulent chaos (i.e., the vortex configurations allowed by the theory) depends directly on the fact that pressure is governed 


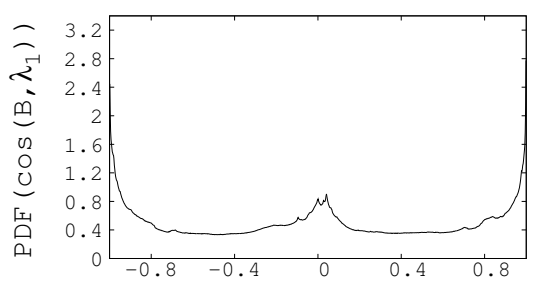

$\cos \left(B, \lambda_{1}\right)$

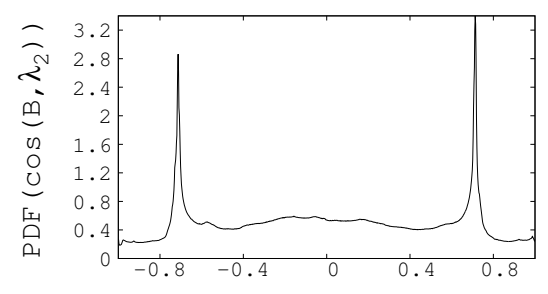

$\cos \left(B, \lambda_{2}\right)$

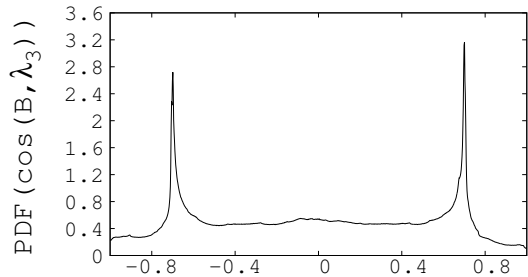

$\cos \left(B, \lambda_{3}\right)$

FIG. 25. Probability density functions of magnetic-field/strain-rate-eigenvector cosines for $N=$ $2.5 \times 10^{3}$ at time $t=58.29 \tau_{\mathrm{d}}$. From left to right: $\cos \left(\mathbf{B}, \boldsymbol{\lambda}_{1}\right), \cos \left(\mathbf{B}, \boldsymbol{\lambda}_{2}\right)$, and $\cos \left(\mathbf{B}, \boldsymbol{\lambda}_{3}\right)$.
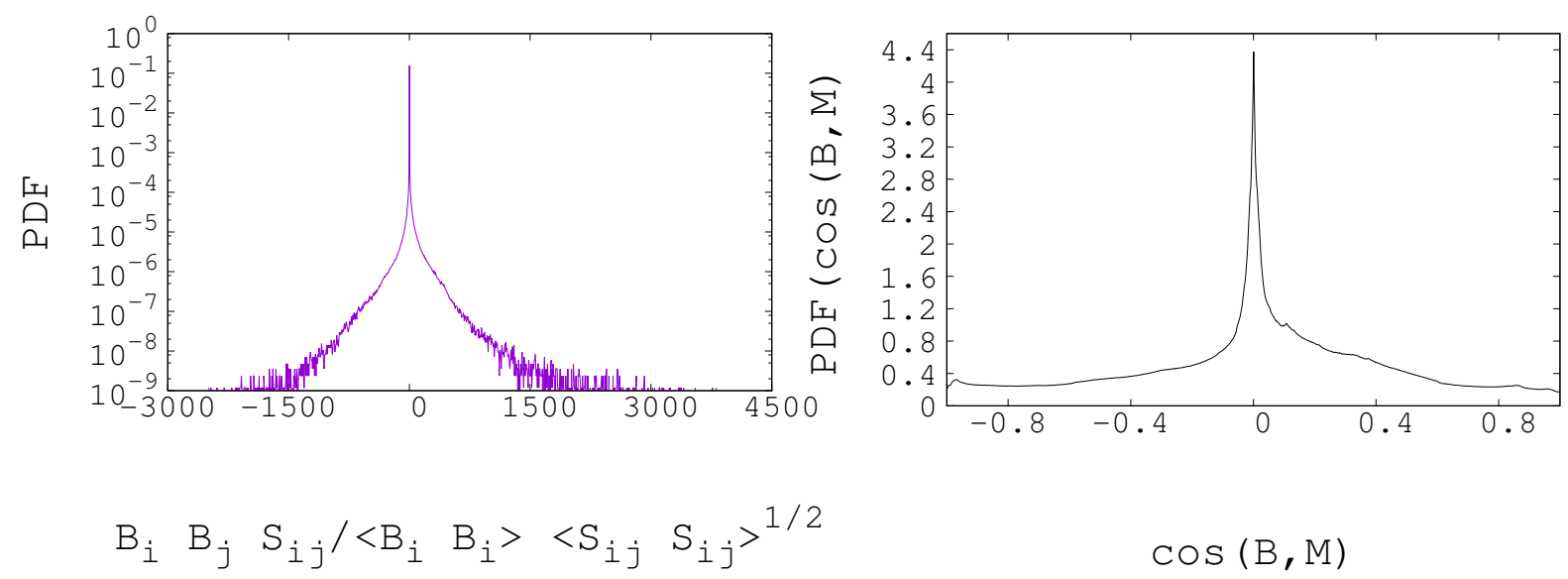

FIG. 26. Left: Probability density function of scaled magnetic-energy source $\frac{\left\langle B_{i} B_{j} S_{i j}\right\rangle}{\left\langle B_{i} B_{i}\right\rangle\left\langle S_{i j} S_{i j}\right\rangle^{1 / 2}}$. Right: Probability density function of magnetic-field/magnetic-field stretching vector cosine $\cos (\mathbf{B}, \mathbf{M})$. Both results are for $N=2.5 \times 10^{3}$ at time $t=58.29 \tau_{\mathrm{d}}$.

by an elliptic equation (action at a distance), albeit with a very rare (and indeed intriguing in classical field theory) nonlinear source. The pressure field cannot form any (localized) structures, and affects vortex structures only indirectly, via its effect on the velocity field. In MHD, one can view the gauge field as another way of interaction with the inertial field, since the gauge field obeys a very different (parabolic) evolution equation than pressure. Hence, it can develop localized structures, and can directly impact upon the vorticity field via the curl of the Lorentz force. This research aimed at enlarging our understanding of these important MHD issues.

It indicates that vorticity/magnetic field interactions are ways of transforming various MHD structures, and even generating new ones. Hence, at small and intermediate interaction numbers, a vortex tube transforms a magnetic ring into a flattened spiral, at intermediate $\mathrm{N}$, the 
Lorentz force generates a vorticity solenoid, and at high $\mathrm{N}$, the effect of the gauge field on the flow results in the generation of new, collocated magnetic and vortex rings and sheets. In all cases, we have indicated the energy dynamics and spectra, showing how the transfer of energy from flow to magnetic degrees of freedom at small and intermediate $\mathrm{N}$, is reversed at high $\mathrm{N}$, and how the generated structures affect the shape of kinetic and magnetic energy spectra. Without doubt, the gauge field appears to be a great transformative/creative force, as far as, the vorticity field is concerned. This is particularly evident in the "serpentine road" to vortex reconnection depicted in the high $\mathrm{N}$ case results.

Another general conclusion supported by the results is that, at small $N$ values, $\boldsymbol{\omega}$ dominates, and $\mathbf{B}$ behaves like a material-field advected by the flow, whilst, at large $N$, the backreaction of $\mathbf{B}$ on $\boldsymbol{\omega}$ is the key dynamical factor, and, as a result, new vortex structures appear that induce magnetic field structures of similar morphology.

It is important to continue placing emphasis on structural aspects of turbulent MHD flows, by either extending the present "synthetic" approach to the interaction between more complicated structural patterns, or/and by performing accompanying "analytic" studies, i.e., detecting and tracking structural elements within an actual turbulent MHD flow field. Incorporating the findings of such studies into the statistical physics modeling of turbulence, is a promising approach for tackling the notorious "turbulence problem".

\section{ACKNOWLEDGEMENTS}

I am very grateful to Tony Leonard and Axel Brandenburg for valuable discussions, and Caltech overall for its generous hospitality during my visiting faculty appointment.

[1] P. Manneville, Dissipative Structures and Weak Turbulence, San Diego: Academic Press (1990).

[2] W.D. McComb, Homogeneous, Isotropic Turbulence: Phenomenology, Renormalization and Statistical Closures, Oxford: Oxford University Press (2014).

[3] C.F. Barenghi, R.J. Donnelly (eds.), Quantized Vortex Dynamics and Superfluid Turbulence, Berlin: Springer (2010). 
[4] R.J. Donnelly, Quantized Vortices in Helium II, Cambridge: Cambridge University Press (2008).

[5] L. Rezzolla, O. Zanotti, Relativistic Hydrodynamics, Oxford: Oxford University Press (2013).

[6] G.L. Eyink, T.D. Drivas, Cascades and Dissipative Anomalies in Relativistic Fluid Turbulence, arXiv:1704.03541 [physics.flu-dyn]

[7] P.A. Davidson, turbulence, Oxford: Oxford University Press (2004).

[8] A. Tsinober, An Informal Introduction to Turbulence, Kluwer Academic Publishers (2001).

[9] I.V. Ovchinnikov, Introduction to Supersymmetric theory of stochastics, arXiv:1511.03393v3 [math-ph]

[10] K. Yagi, T. Hatsuda, Y. Miake, Quark-Gluon Plasma, Cambridge: Cambridge University Press (2008).

[11] S. Mrowczynski, M.H. Thoma, What do Electromagnetic plasmas tell us about the QuarkGluon Plasma? Annu. Rev. Nucl. Part. Sci. 57, 61-94 (2007).

[12] S. Galtier, Introduction to modern magnetohydrodynamics, Cambridge: Cambridge University Press (2016).

[13] E. Guyon, J.-P. Hulin, L. Petit, C.D. Mitescu, Physical Hydrodynamics, Oxford: Oxford University Press (2015).

[14] A.A.R. Townsend, The Structure of Turbulent Shear Flow, Cambridge: Cambridge University Press (1975).

[15] D.I. Pulin, P.G. Saffman, Vortex dynamics in turbulence, Annu. Rev. Fluid Mech. 30, 31-51 (1998).

[16] D. Kivotides, A. Leonard, Quantized turbulence physics, Phys. Rev. Lett. 90, 234503 (2003).

[17] D. Kivotides, A. Leonard, Geometrical physics of the many vortex filament problem, EPL 66, 6975 (2004).

[18] D. Biskamp, Magnetohydrodynamic turbulence, Cambridge: Cambridge University Press (2003).

[19] H. Aluie, G.L. Eyink, Scale Locality of Magnetohydrodynamic Turbulence, Phys. Rev. Lett. 104, 081101 (2010).

[20] A. Nordlund, A. Brandenburg, R.L. Jennings, M. Rieutord, J. Ruokolainen, R.F. Stein, I. Tuominen, Dynamo action in stratified convection with overshoot, Astrophys. J. 392, 647-652 (1992). 
[21] D. Kivotides, A.J. Mee, C.F. Barenghi, Magnetic field generation by coherent turbulence structures, New Journal of Physics 9, 291-299 (2007).

[22] A. Brandenburg, I. Procaccia, D. Segel, The size and dynamics of magnetic flux structures in magnetohydrodynamic turbulence, Physics of Plasmas 2, 1148-1156 (1995).

[23] J.I. Sakai, K. Nishi, I.V. Sokolov, A model of a single-loop flare: disruption of a magnetic flux tube driven by collision of two moving solitary magnetic kinks, Astrophys. J. 576, 519-532 (2002).

[24] F. Del Sordo, S. Candelaresi, A. Brandenburg, Magnetic-field decay of three interlocked flux rings with zero linking number, Phys. Rev. E 81, 036401 (2010).

[25] S. Candelaresi, A. Brandenburg, Decay of helical and non-helical magnetic knots, Phys. Rev. E 84, 016406 (2011).

[26] V. Carbone, P. Veltri, A. Mangeney, Coherent structure formation and magnetic field line reconnection in magnetohydrodynamic turbulence, Phys. Fluids A 2, 1487-1496 (1990).

[27] E.L. Rempel, A.C.-L. Chian, A. Brandenburg, P.R. Munoz, S.C. Shadden, Coherent structures and the saturation of a nonlinear dynamo, J. Fluid Mech. 729, 309-329 (2013).

[28] Y. Yang, W.H. Matthaeus, Y. Shi, M. Wan, S. Chen, Compressibility effect on coherent structures, energy transfer, and scaling in magnetohydrodynamic turbulence, Phys. Fluids 29, 035105 (2017).

[29] K. Arzner, B. Knaepen, D. Carati, N. Denewet, L. Vlahos, The effect of coherent structures on stochastic acceleration in MHD turbulence, Astrophys. J. 637, 322-332 (2006).

[30] V. Zhdankin, S. Boldyrev, J.C. Perez, S.M. Tobias, Energy Dissipation in Magnetohydrodynamic Turbulence: Coherent structures or "Nanoflares"? arXiv:1409.3285vl [astro-ph.SR].

[31] R.S. Miller, F. Mashayek, V. Adumitroaie, P. Givi, Structure of homogeneous nonhelical magnetohydrodynamic turbulence, Phys. Plasmas 3, 3304-3317 (1996).

[32] A.A. Schekochihin, J.L. Maron, S.C. Cowley, J.C. McWilliams, The small-scale structure of magnetohydrodynamic turbulence with large magnetic Prandtl numbers, Astrophys. J. 576, 806-813 (2002).

[33] D.A. Gurnett, A. Bhattacharjee, Introduction to Plasma Physics, Cambridge: Cambridge University Press (2017).

[34] J.-Z. Wu, H.-Y. Ma, M.-D. Zhou, Vorticity and Vortex Dynamics, Berlin: Springer (2006). 
[35] S.M. Mahajan, Z. Yoshida, Relativistic generation of vortex and magnetic field, Phys. Plasmas 18, 055701 (2011).

[36] V. Arnold, B. Khesin, Topological Methods in Hydrodynamics, Berlin: Springer (1998).

[37] F.H. Harlow, J.E. Welch, Numerical Calculation of Time-Dependent Viscous Incompressible Flow of Fluid with Free Surface, Phys. Fluids 8, 2182-2189 (1965).

[38] J.L. Guermond, P. Minev, J. Shen, An overview of projection methods for incompressible flows, Comp. Meth. Appl. Mech. Eng. 195, 6011-6045 (2006).

[39] P. Orlandi, Fluid Flow Phenomena: A numerical Toolkit, Berlin: Springer (2002).

[40] A. Leonard, Nonlocal theory of area-varying waves on axisymmetric vortex tubes, Phys. Fluids 6, 765 (1994).

[41] S. Molokov et al. (eds.), Magnetohydrodynamics - Historical Evolution and Trends, Berlin: Springer (2007).

[42] T.S. Lund, M.M. Rogers, An improved measure of strain state probability in turbulent flows, Phys. Fluids 6, 1838-1847 (1994).

[43] S.K. Nemirovskii, Energy spectrum of the 3D velocity field, induced by vortex tangle, J. Low Temp. Phys. 171, 504-510 (2013). 